Sharif University of Technology
Scientia Iranica
Transactions E: Industrial Engineering
http://scientiairanica.sharif.edu
IRAN I CA

\title{
Adjustable approaches to multi-criteria group decision making based on inverse fuzzy soft matrices
}

\author{
S. Petchimuthu ${ }^{\mathrm{a}, *}$ and H. Kamaci ${ }^{\mathrm{b}}$ \\ a. Department of Mathematics, University College of Engineering, Nagercoil-629004, Tamilnadu, India. \\ b. Department of Mathematics, Faculty of Science and Arts, Yozgat Bozok University, Yozgat, Turkey. \\ Received 23 August 2019; received in revised form 14 June 2020; accepted 21 September 2020
}

\author{
KEYWORDS \\ Fuzzy sets; \\ Inverse fuzzy soft sets; \\ Inverse fuzzy soft \\ matrices; \\ Operations of inverse \\ fuzzy soft matrices; \\ Multi-criteria group \\ decision making.
}

\begin{abstract}
In this paper, we focus on the matrices representing the inverse fuzzy soft sets over both the universal object set and the universal parameter set. Some basic operations and properties of these inverse fuzzy soft matrices were investigated. Moreover, two adjustable approaches to Multi-Criteria Group Decision Making (MCGDM), namely Inverse Fuzzy Soft Sum-Product Decision Making (IFSSPDM) and Inverse Fuzzy Soft Distributive If-difference Decision Making (IFSDIf-dDM), were developed. The IFSSPDM approach achieved the optimal choice for the MCGDM problem based on the inverse fuzzy soft structures consisting of multiple-discrete parameter sets and common universal object sets. The objective of IFSDIf-dDM approach was to present a solution to the MCGDM problem based on the inverse fuzzy soft structures consisting of a common universal parameter set and two discrete universal object sets. Thus, the solutions could be obtained using the practicality of inverse fuzzy soft matrices for two different types of decision making problems. Besides, the comparisons made showed that the proposed approaches produced more convincing outputs than the current fuzzy soft approaches.
\end{abstract}

(C) 2022 Sharif University of Technology. All rights reserved.

\section{Introduction}

To deal with the foremost necessary origin background of problems containing uncertainty and obscurity, the fuzzy and soft sets were introduced by Zadeh [1] in 1965 and Molodtsov [2] in 1999, respectively. The authors in [3-6] concentrated on the soft sets and their operations to solve day-to-day life decision making problems, which involved different kinds of uncertainties. Especially, Çag̃man and Enginog̃lu [7] redefined the operations of Molodtsov's soft sets to make it more

*. Corresponding author.

E-mail addresses: svmuthu1983@gmail.com (S.

Petchimuthu); huseyin.kamaci@hotmail.com (H. Kamacı)

doi: $10.24200 /$ sci.2020.54294.3686 functional and then, adapted them to the soft matrices in [8]. The soft matrix theory was further improved by the researchers [9-14] towards the applications of decision making problems.

In 2001, Maji et al. [15] introduced a fuzzy soft set by combining Molodtsov's soft set and Zadeh's fuzzy set. This set can be considered as a hybridization of a soft set and fuzzy set. The theory of fuzzy soft set has turned into a center of attraction among research scholars because it can be applied more effectively to decision making, game theory, pattern recognition, medical diagnosis, etc. Many researchers endeavored to develop outstanding algorithms/methods/procedures to deal with decision making problems [16-22], medical diagnosis problems [23-26], and job requirement problems [27] under fuzzy soft environment. In 2012, Çağman and Enginog̃lu [28] derived the matrix repre- 
sentations matching the fuzzy soft sets and discussed their natural implementations. The fuzzy soft matrix, a new way of approaching the fuzzy soft set, is more practical and useful. Therefore, some authors [29-32] adopted these matrix notations to formulate methods that aim to find optimal solutions to Multi-Criteria Group Decision Making (MCGDM) problems.

In recent years, two studies have been presented on the inverse structure of fuzzy soft sets from different perspectives. In 2016, Çetkin et al. [33] initiated the idea of inverse fuzzy soft sets on the common universal set and then, Khalil and Hassan [34] introduced the inverse fuzzy soft sets on the common parameter set in 2019. They focused on the advantages of these inverse fuzzy soft sets in practice and endeavored to reinforce this argument for decision making. It is known that the matrices corresponding to the fuzzy soft sets are both more practical and time-saving. Relatedly, it is important to represent both types of inverse structures of fuzzy soft sets through matrices.

The main objective of this study is to create the matrix representations of inverse fuzzy soft sets introduced by Çetkin et al. [33] and Khalil and Hassan [34]. Besides, it is aimed to propose new approaches to the MCGDM based on (inverse) fuzzy soft information and to create an outstanding decision making model that can achieve optimal choice when the number of universal object sets is two. The contributions of this paper are detailed as follows:

- The inverse fuzzy soft matrices are theorized. These matrices offer the opportunity to represent both the concept of inverse fuzzy soft set proposed by Çetkin et al. [33] and the concept of inverse fuzzy soft set proposed by Khalil and Hassan [34], thereby to perform two-way evaluations. Moreover, some inverse fuzzy soft matrix $r$-products and $c$-products such as and, or, exclusive or, difference, if, and iff are derived. The properties and relations of these products are itemized;

- An adjustable approach to MCGDM is proposed under the (inverse) fuzzy soft environment. The evaluation bases of the new approach are multiple. This model is compared with some of the existing fuzzy soft decision making models and thus, its advantages are revealed;

- The number of parameter sets in decision making can be multiple and many fuzzy soft decision making models have been proposed to deal with such problems. However, all the existing models become insufficient as the number of universal sets increases. Algorithm 2 is proposed to eliminate the restriction on the number of universal object sets in decision making. This offers a different perspective to (inverse) fuzzy soft decision making.
The remainder of this paper is organized as follows. In the second section, some preliminary fundamental definitions are stated. The third section introduces the matrices matching the inverse fuzzy soft sets and their related operations. In the fourth section, an MCGDM model is organized to obtain an optimum universal object set concerning the multiple-discrete parameter sets under the inverse fuzzy soft environment. Besides, the efficiency and performance of the proposed model are analyzed with examples and comparisons. The fifth section is devoted to solving the MCGDM problem based on the inverse fuzzy soft structures consisting of a common universal parameter set and two discrete universal object sets. The last section consists of the conclusion of the paper.

\section{Preliminaries}

In this section, we firstly retrospect the notions of fuzzy set, fuzzy soft set, and inverse fuzzy soft set.

Definition 1 [1]. Let $O$ be a set of objects. A fuzzy set $A$ in $O$ is defined as:

$$
A=\left\{\left(o_{j}, \mu_{A}\left(o_{j}\right)\right) / o_{j} \in O\right\}
$$

where $\mu_{A}: O \rightarrow[0,1]$ is a function. Also, the function $\mu_{A}$ is called the membership function and $\mu_{A}\left(o_{j}\right)$ is called the membership value for each $o_{j} \in O$.

Definition 2 [15]. Let $O$ be a set of objects and $X$ be a set of parameters. Also, let $\mathscr{P}(O)$ denote the set of all fuzzy sets over $O$. Then, the following set of ordered pairs:

$$
\Psi_{X}^{O}=\left\{\left(x_{i}, \psi\left(x_{i}\right)\right): x_{i} \in X, \psi\left(x_{i}\right) \in \mathscr{P}(O)\right\},
$$

is called a fuzzy soft set over $O$, where $\psi: X \rightarrow \mathscr{P}(O)$ is a set-valued mapping.

In the above definition, we remark that $\psi\left(x_{i}\right)$ is a fuzzy set for each $x_{i} \in X$. Assume that $\psi_{x_{i}}$ is the membership function for the fuzzy set $\psi\left(x_{i}\right)$. Clearly, $\psi_{x_{i}}\left(o_{j}\right)$ is the membership value for each $o_{j} \in O$. Therefore, for each $x_{i} \in X$, the corresponding fuzzy set $\psi\left(x_{i}\right)$ can be written as $\psi\left(x_{i}\right)=\left\{\left(o_{j}, \psi_{x_{i}}\left(o_{j}\right)\right) / o_{j} \in O\right\}$.

Example 1. Let $O=\left\{o_{1}, o_{2}, o_{3}, o_{4}\right\}$ be a set of objects and $X=\left\{x_{1}, x_{2}, x_{3}\right\}$ be a set of parameters. If we define:

$$
\begin{aligned}
& \psi: X \rightarrow \mathscr{P}(O) \text { by } \\
& \psi\left(x_{1}\right)=\left\{\left(o_{1}, 1\right),\left(o_{2}, 0.6\right),\left(o_{3}, 0.8\right),\left(o_{4}, 0.2\right)\right\}, \\
& \psi\left(x_{2}\right)=\left\{\left(o_{1}, 0.1\right),\left(o_{2}, 0.5\right),\left(o_{3}, 0\right),\left(o_{4}, 0.1\right)\right\}, \\
& \psi\left(x_{3}\right)=\left\{\left(o_{1}, 0.4\right),\left(o_{2}, 0.6\right),\left(o_{3}, 0\right),\left(o_{4}, 0\right)\right\},
\end{aligned}
$$

then we have the following fuzzy soft set: 


$$
\Psi_{X}^{O}=\left\{\begin{array}{l}
\left(x_{1},\left\{\left(o_{1}, 1\right),\left(o_{2}, 0.6\right),\left(o_{3}, 0.8\right),\left(o_{4}, 0.2\right)\right\}\right), \\
\left(x_{2},\left\{\left(o_{1}, 0.1\right),\left(o_{2}, 0.5\right),\left(o_{3}, 0\right),\left(o_{4}, 0.1\right)\right\}\right), \\
\left(x_{3},\left\{\left(o_{1}, 0.4\right),\left(o_{2}, 0.6\right),\left(o_{3}, 0\right),\left(o_{4}, 0\right)\right\}\right)
\end{array}\right\} .
$$

In the literature, there are two different types of inverse fuzzy soft sets. The first of these is the concept of inverse fuzzy soft set on the universal object set $O$, which was defined by Ceetkin et al. [33] in 2016, and the other one is the idea of the inverse fuzzy soft set on the universal parameter set $X$, which was introduced by Khalil and Hassan [34] in 2019. These emerging approaches are different from each other. Also, the operations derived from these inverse fuzzy soft sets have different characteristics.

Definition 3 [33]. Let $O$ be a set of objects and $X_{c}$ be a set of parameters. Also, let $\mathscr{P}\left(X_{c}\right)$ denote the set of all fuzzy sets over $X_{c}$. Then, the following set of ordered pairs:

$$
\widetilde{\Psi}_{X_{c}}^{O}=\left\{\left(o_{j}, \widetilde{\psi}\left(o_{j}\right)\right): o_{j} \in O, \widetilde{\psi}\left(o_{j}\right) \in \mathscr{P}\left(X_{c}\right)\right\}
$$

is called an inverse fuzzy soft set over $O$, where $\psi$ : $O \rightarrow \mathscr{P}\left(X_{c}\right)$ is a set-valued mapping.

Definition 4 [34]. Let $O_{r}$ be a set of objects and $X$ be a set of parameters. Also, let $\mathscr{P}(X)$ denote the power set of $X$. Then, the following set of ordered pairs:

$$
\widetilde{\Psi}_{O_{r}}^{X}=\left\{\left(o_{j}^{r}, \widetilde{\psi}\left(o_{j}^{r}\right)\right): o_{j}^{r} \in O_{r}, \widetilde{\psi}\left(o_{j}^{r}\right) \in \mathscr{P}(X)\right\}
$$

is called an inverse fuzzy soft set over $X$, where $\widetilde{\psi}$ : $O_{r} \rightarrow \mathscr{P}(X)$ is a set-valued mapping.

To understand the difference between fuzzy soft set on $O$ and inverse fuzzy soft set on $O$, we can consider the following example.

Assume that a company plans to recruit personnel for an empty position. Suppose that there are five candidate men who have applied for this empty position. For this purpose, the company management determines an expert team $E_{1}$ (consisting of men) and wants the team to evaluate these candidates according to some parameters. The expert team $E_{1}$ interviews each of the candidates and, as a result, can create a fuzzy soft set. In other words, the expert team $E_{1}$ thinks "which candidates have the attribute (parameter) $x$ ?" If the number of candidates (men) is 90 , then it is very difficult for the expert team $E_{1}$ to achieve an answer to this question. For instance, it is difficult to determine candidates (with fuzzy values) having the parameter " $x$-proper diction" from 90 candidates in a snap. However, the expert team $E_{1}$ interviewing a candidate can determine all attributes (parameters) in the (fuzzy) parameter set for this candidate. In other words, the expert team $E_{1}$ thinks that "what are the attributes (parameters) in the universal parameter set for each candidate $o$ ?" and then, they can create an inverse fuzzy soft set.

The above problem reveals the difference between inverse fuzzy soft set over the universal set $O$ and inverse soft set over the parameter $X$.

Now, suppose that four female candidates have applied for this empty position. There is an expert team $E_{2}$ (consisting of women) to evaluate these candidates according to the parameters. The expert team $E_{2}$ can create an inverse fuzzy soft set for the four female candidates. The inverse fuzzy soft sets formed by the two expert teams $E_{1}$ and $E_{2}$ include the evaluation of candidates in two different universal sets (one of which is a set of five male candidates and the other is a set of four female candidates) under the same parameter set $X$. Consequently, an inverse fuzzy soft set is obtained for five male candidates and an inverse fuzzy soft set for four female candidates over the parameter set $X$.

The following example better explains this difference.

\section{Example 2.}

(i) Let $O_{1}=\left\{o_{1}^{1}, o_{2}^{1}, o_{3}^{1}\right\}$ be a set of objects. Also, let us take the parameter sets as $X_{1}=\left\{x_{1}^{1}, x_{2}^{1}\right\}$ and $X_{2}=\left\{x_{1}^{2}, x_{2}^{2}, x_{3}^{2}\right\}$. Then, we can write two inverse fuzzy soft sets over $O_{1}$ with respect to the sets $X_{1}$ and $X_{2}$ as follows:

$$
\begin{aligned}
& \widetilde{\Psi}_{X_{1}}^{O_{1}}=\{\left(o_{1}^{1},\left\{\left(x_{1}^{1}, 0.5\right),\left(x_{2}^{1}, 0.6\right)\right\}\right), \\
&\left(o_{2}^{1},\left\{\left(x_{1}^{1}, 0.9\right),\left(x_{2}^{1}, 1\right)\right\}\right), \\
&\left.\left(o_{3}^{1},\left\{\left(x_{1}^{1}, 0.4\right),\left(x_{2}^{1}, 0.7\right)\right\}\right)\right\} \\
& \widetilde{\Psi}_{X_{2}}^{O_{1}}=\left\{\left(o_{1}^{1},\left\{\left(x_{1}^{2}, 0.6\right),\left(x_{2}^{2}, 0.3\right),\left(x_{3}^{2}, 0.4\right)\right\}\right),\right. \\
&\left(o_{2}^{1},\left\{\left(x_{1}^{2}, 0.8\right),\left(x_{2}^{2}, 0.9\right),\left(x_{3}^{2}, 0.4\right)\right\}\right), \\
&\left.\left(o_{3}^{1},\left\{\left(x_{1}^{2}, 0.1\right),\left(x_{2}^{2}, 0.2\right),\left(x_{3}^{2}, 0.7\right)\right\}\right)\right\} .
\end{aligned}
$$

(ii) Let $O_{1}=\left\{o_{1}^{1}, o_{2}^{1}, o_{3}^{1}\right\}, O_{2}=\left\{o_{1}^{2}, o_{2}^{2}, o_{3}^{2}\right\}$, and $O_{3}=$ $\left\{o_{1}^{3}, o_{2}^{3}\right\}$ be three different sets of objects. Also, let us take the parameter set as $X_{1}=\left\{x_{1}^{1}, x_{2}^{1}\right\}$. Then, we can write three inverse fuzzy soft sets over $X$ with respect to the sets $O_{1}, O_{2}$, and $O_{3}$ as follows:

$$
\begin{aligned}
\widetilde{\Psi}_{O_{1}}^{X_{1}}=\{ & \left(o_{1}^{1},\left\{\left(x_{1}^{1}, 0.4\right),\left(x_{2}^{1}, 0.6\right)\right\}\right), \\
& \left(o_{2}^{1},\left\{\left(x_{1}^{1}, 0.9\right),\left(x_{2}^{1}, 0.7\right)\right\}\right), \\
& \left.\left(o_{3}^{1},\left\{\left(x_{1}^{1}, 0.3\right),\left(x_{2}^{1}, 0.5\right)\right\}\right)\right\},
\end{aligned}
$$




$$
\begin{aligned}
& \widetilde{\Psi}_{O_{2}}^{X_{1}}=\{(\left(o_{1}^{2},\left\{\left(x_{1}^{1}, 0.4\right),\left(x_{2}^{1}, 0.6\right)\right\}\right), \\
&\left(o_{2}^{2},\left\{\left(x_{1}^{1}, 0.8\right),\left(x_{2}^{1} 0.6\right)\right\}\right), \\
&\left.\left(o_{3}^{2},\left\{\left(x_{1}^{1}, 0.3\right),\left(x_{2}^{1}, 0.5\right)\right\}\right)\right\}, \\
& \widetilde{\Psi}_{O_{3}}^{X_{1}}=\left\{\left(o_{1}^{3},\left\{\left(x_{1}^{1}, 0.2\right),\left(x_{2}^{1}, 0.7\right)\right\}\right),\right. \\
&\left.\left(o_{2}^{3},\left\{\left(x_{1}^{1}, 0.3\right),\left(x_{2}^{1}, 0.1\right)\right\}\right)\right\} .
\end{aligned}
$$

In Example 2, the parameter sets $X_{1}, X_{2}$ and universal (object) sets $\mathrm{O}_{1}, \mathrm{O}_{2}$, and $\mathrm{O}_{3}$ can be discrete sets.

\section{Inverse fuzzy soft matrices with operations}

Up to the present, many researchers have been interested in matrix representations of fuzzy soft sets on $O$. In this section, we define the inverse fuzzy soft sets (given in Definitions 3 and 4 ) representing the inverse fuzzy soft sets on $O$ and $X$. Thereby, we will address the matrices matching the inverse fuzzy soft sets described not only on $O$, but also on $X$.

Definition 5. Let $O_{r}=\left\{o_{j}^{r}: j \in J\right\}$ and $X_{c}=\left\{x_{i}^{c}\right.$ : $i \in I\}$, where $I$ and $J$ are the index sets. Then, the inverse fuzzy soft matrix for the inverse fuzzy soft set $\left(\widetilde{\Psi}_{X_{c}}^{O_{r}}\right.$ or $\left.\widetilde{\Psi}_{O_{r}}^{X_{c}}\right)$ is $\left[a_{i j}^{(c, r)}\right]$, where for all $i$ and $j$ :

$$
a_{i j}^{(c, r)}= \begin{cases}\widetilde{\psi}_{o_{j}^{r}}\left(x_{i}^{c}\right), & \text { if }\left(x_{i}^{c}, \widetilde{\psi}_{o_{j}^{r}}\left(x_{i}^{c}\right)\right) \in \widetilde{\psi}\left(o_{j}^{r}\right) \\ 0, & \text { otherwise }\end{cases}
$$

Example 3. The inverse fuzzy soft matrices representing the inverse fuzzy soft sets $\widetilde{\Psi}_{X_{1}}^{O_{1}}$ and $\widetilde{\Psi}_{X_{2}}^{O_{1}}$ given in Example 2 (i) are respectively:

$$
\left[a_{i j}^{(1,1)}\right]=\left[\begin{array}{ccc}
0.5 & 0.9 & 0.4 \\
0.6 & 1 & 0.7
\end{array}\right]
$$

and

$$
\left[a_{m j}^{(2,1)}\right]=\left[\begin{array}{lll}
0.6 & 0.8 & 0.1 \\
0.3 & 0.9 & 0.2 \\
0.4 & 0.4 & 0.7
\end{array}\right]
$$

The inverse fuzzy soft matrices representing the inverse fuzzy soft sets $\widetilde{\Psi}_{O_{1}}^{X_{1}}, \widetilde{\Psi}_{O_{2}}^{X_{1}}$, and $\widetilde{\Psi}_{O_{3}}^{X_{1}}$ given in Example 2 (ii) are respectively:

$$
\begin{gathered}
{\left[b_{i j}^{(1,1)}\right]=\left[\begin{array}{lll}
0.4 & 0.9 & 0.3 \\
0.6 & 0.7 & 0.5
\end{array}\right],} \\
{\left[b_{i k}^{(1,2)}\right]=\left[\begin{array}{lll}
0.4 & 0.8 & 0.3 \\
0.6 & 0.6 & 0.5
\end{array}\right],}
\end{gathered}
$$

and

$$
\left[b_{i l}^{(1,3)}\right]=\left[\begin{array}{ll}
0.2 & 0.3 \\
0.7 & 0.1
\end{array}\right]
$$

Notation 1. There is an exact matching between the inverse fuzzy soft matrices and the inverse fuzzy soft sets. $\operatorname{IFSM}(O)$ indicates the set of all inverse fuzzy soft matrices on the common universal set $O$. Likewise, $\operatorname{IFSM}(X)$ indicates the set of all inverse fuzzy soft matrices on the common parameter set $X$.

Notation 2. If $|X|=t$ and $|O|=v$, then the set of all $t \times v$ inverse fuzzy soft matrices on $O$ and $X$ is characterized by $I F S M_{t \times v}$.

Considering Example 3, it is seen that $\left[a_{i j}^{(1,1)}\right]$, $\left[a_{m j}^{(2,1)}\right] \in \operatorname{IFSM}\left(O_{1}\right)$ and $\left[b_{i j}^{(1,1)}\right], \quad\left[b_{i k}^{(1,2)}\right], \quad\left[b_{i l}^{(1,3)}\right] \in$ $\operatorname{IFSM}\left(X_{1}\right)$. On the other hand, it is clear that $\left[a_{i j}^{(1,1)}\right]$, $\left[b_{i j}^{(1,1)}\right],\left[b_{i k}^{(1,2)}\right] \in I F S M_{2 \times 3},\left[a_{m j}^{(2,1)}\right] \in I F S M_{3 \times 3}$ and $\left[b_{i l}^{(1,3)}\right] \in \operatorname{IFSM}_{2 \times 2}$. We have $\left[a_{i j}^{(1,1)}\right],\left[b_{i j}^{(1,1)}\right] \in \operatorname{IFSM}_{2 \times 3}$ while $\left[a_{i j}^{(1,1)}\right] \in \operatorname{IFSM}\left(O_{1}\right)$ and $\left[b_{i j}^{(1,1)}\right] \in \operatorname{IFSM}\left(X_{1}\right)$. The result is evident because these two matrices are constructed using the sets $O_{1}$ and $X_{1}$. However, it is worth mentioning that $\left[a_{i j}^{(1,1)}\right],\left[b_{i k}^{(1,2)}\right] \in I F S M_{2 \times 3}$ while $\left[a_{i j}^{(1,1)}\right] \in \operatorname{IFSM}\left(O_{1}\right)$ and $\left[b_{i k}^{(1,3)}\right] \in \operatorname{IFSM}\left(X_{1}\right)$.

Definition 6. Let $\left[a_{i j}^{\left(c_{1}, r_{1}\right)}\right],\left[b_{i j}^{\left(c_{2}, r_{2}\right)}\right] \in I F S M_{t \times v}$ :

a) If $c_{1}=c_{2}=c$ and $a_{i j}^{\left(c, r_{1}\right)} \leq b_{i j}^{\left(c, r_{2}\right)}$ for all $i, j$, then the matrix $\left[a_{i j}^{\left(c, r_{1}\right)}\right]$ is termed a column-inverse fuzzy submatrix ( $c$-inverse fuzzy soft submatrix) of $\left[b_{i j}^{\left(c, r_{2}\right)}\right]$. This is symbolically represented by $\left[a_{i j}^{\left(c, r_{1}\right)}\right] \sqsubseteq_{c}\left[b_{i j}^{\left(c, r_{2}\right)}\right]$;

b) If $r_{1}=r_{2}=r$ and $a_{i j}^{\left(c_{1}, r\right)} \leq b_{i j}^{\left(c_{2}, r\right)}$ for all $i, j$, then the matrix $\left[a_{i j}^{\left(c_{1}, r\right)}\right]$ is termed a row-inverse fuzzy soft submatrix ( $r$-inverse fuzzy soft submatrix) of $\left[b_{i j}^{\left(c_{2}, r\right)}\right]$. This is symbolically represented by $\left[a_{i j}^{\left(c_{1}, r\right)}\right] \sqsubseteq_{r}\left[b_{i j}^{\left(c_{2}, r\right)}\right]$;

c) If $c_{1}=c_{2}=c, r_{1}=r_{2}=r$ and $a_{i j}^{(c, r)} \leq b_{i j}^{(c, r)}$ for all $i, j$, then the matrix $\left[a_{i j}^{(c, r)}\right]$ is termed an inverse fuzzy soft submatrix of $\left[b_{i j}^{(c, r)}\right]$. This is symbolically represented by $\left[a_{i j}^{(c, r)}\right] \sqsubseteq\left[b_{i j}^{(c, r)}\right]$.

Definition 7. Let $\left[a_{i j}^{\left(c_{1}, r_{1}\right)}\right],\left[b_{i j}^{\left(c_{2}, r_{2}\right)}\right] \in I F S M_{t \times v}$ :

a) If $c_{1}=c_{2}=c$ and $a_{i j}^{\left(c, r_{1}\right)}=b_{i j}^{\left(c, r_{2}\right)}$ for all $i, j$, then the matrices $\left[a_{i j}^{\left(c, r_{1}\right)}\right]$ and $\left[b_{i j}^{\left(c, r_{2}\right)}\right]$ are termed column-inverse fuzzy soft equal matrices (c-inverse fuzzy soft equal matrices). This is symbolically represented by $\left[a_{i j}^{\left(c, r_{1}\right)}\right]={ }_{c}\left[b_{i j}^{\left(c, r_{2}\right)}\right]$;

b) If $r_{1}=r_{2}=r$ and $a_{i j}^{\left(c_{1}, r\right)}=b_{i j}^{\left(c_{2}, r\right)}$ for all $i, j$, then the matrices $\left[a_{i j}^{\left(c_{1}, r\right)}\right]$ and $\left[b_{i j}^{\left(c_{2}, r\right)}\right]$ are termed row-inverse fuzzy soft equal matrices ( $r$-inverse fuzzy soft equal matrices). This is symbolically represented by $\left[a_{i j}^{\left(c_{1}, r\right)}\right]={ }_{r}\left[b_{i j}^{\left(c_{2}, r\right)}\right]$; 
c) If $c_{1}=c_{2}=c, r_{1}=r_{2}=r$ and $a_{i j}^{(c, r)}=b_{i j}^{(c, r)}$ for all $i, j$, then the matrices $\left[a_{i j}^{(c, r)}\right]$ and $\left[b_{i j}^{(c, r)}\right]$ are termed to be inverse fuzzy soft equal matrices. This is symbolically represented by $\left[a_{i j}^{(c, r)}\right]=\left[b_{i j}^{(c, r)}\right]$.

Definition 8. Let $\left[a_{i j}^{(c, r)}\right] \in I F S M_{t \times v}$. Then, the complement to inverse fuzzy soft matrix $\left[a_{i j}^{(c, r)}\right]$ is defined as $\left[a_{i j}^{(c, r)}\right]^{\circ}=\left[d_{i j}^{(c, r)}\right]$, where $d_{i j}^{(c, r)}=1-a_{i j}^{(c, r)}$ for all $i$ and $j$.

Example 4. Let us consider the inverse fuzzy soft matrices given in Example 3. Then, we have $\left[b_{i j}^{(1,1)}\right] \sqsubseteq$ $\left[a_{i j}^{(1,1)}\right],\left[b_{i k}^{(1,2)}\right] \sqsubseteq_{c}\left[b_{i j}^{(1,1)}\right]$ and $\left[b_{i k}^{(1,2)}\right] \sqsubseteq_{c}\left[a_{i j}^{(1,1)}\right]$. Also, we obtain:

$$
\begin{aligned}
& {\left[a_{m j}^{(2,1)}\right]^{\circ}=\left[\begin{array}{lll}
0.4 & 0.2 & 0.9 \\
0.7 & 0.1 & 0.8 \\
0.6 & 0.6 & 0.3
\end{array}\right],} \\
& {\left[b_{i j}^{(1,1)}\right]^{\circ}=\left[\begin{array}{lll}
0.6 & 0.1 & 0.7 \\
0.4 & 0.3 & 0.5
\end{array}\right],} \\
& {\left[b_{i l}^{(1,3)}\right]^{\circ}=\left[\begin{array}{ll}
0.8 & 0.7 \\
0.3 & 0.9
\end{array}\right] .}
\end{aligned}
$$

Definition 9. Let $\left[a_{i j}^{\left(c_{1}, r_{1}\right)}\right],\left[b_{i j}^{\left(c_{2}, r_{2}\right)}\right] \in I F S M_{t \times v}$. Then:

a) The column-intersection (c-intersection) of inverse fuzzy soft matrices $\left[a_{i j}^{\left(c, r_{1}\right)}\right]$ and $\left[b_{i j}^{\left(c, r_{2}\right)}\right]$ for $c_{1}=c_{2}=$ $c$ is defined as $\left[a_{i j}^{\left(c, r_{1}\right)}\right] \Pi_{c}\left[b_{i j}^{\left(c, r_{2}\right)}\right]=\left[d_{i j}\right] \in I F S M_{t \times v}$ where $d_{i j}=\min \left\{a_{i j}^{\left(c, r_{1}\right)}, b_{i j}^{\left(c, r_{2}\right)}\right\}$ for all $i$ and $j$;

(b) The row-intersection ( $r$-intersection) of inverse fuzzy soft matrices $\left[a_{i j}^{\left(c_{1}, r\right)}\right]$ and $\left[b_{i j}^{\left(c_{2}, r\right)}\right]$ for $r_{1}=r_{2}$ $=r$ is defined as $\left[a_{i j}^{\left(c_{1}, r\right)}\right] \Pi_{r}\left[b_{i j}^{\left(c_{2}, r\right)}\right]=\left[d_{i j}\right] \in$ $I F S M_{t \times v}$ where $d_{i j}=\min \left\{a_{i j}^{\left(c_{1}, r\right)}, b_{i j}^{\left(c_{2}, r\right)}\right\}$ for all $i$ and $j$;

(c) The intersection of inverse fuzzy soft matrices $\left[a_{i j}^{(c, r)}\right]$ and $\left[b_{i j}^{(c, r)}\right]$ for $c_{1}=c_{2}=c$ and $r_{1}=r_{2}=r$ is defined as $\left[a_{i j}^{(c, r)}\right] \sqcap\left[b_{i j}^{(c, r)}\right]=\left[d_{i j}\right] \in \operatorname{IFSM}_{t \times v}$ where $d_{i j}=\min \left\{a_{i j}^{(c, r)}, b_{i j}^{(c, r)}\right\}$ for all $i$ and $j$.

Definition 10. Let $\left[a_{i j}^{\left(c_{1}, r_{1}\right)}\right],\left[b_{i j}^{\left(c_{2}, r_{2}\right)}\right] \in I S F M_{t \times v}$ :

a) The column-union (c-union) of inverse fuzzy soft matrices $\left[a_{i j}^{\left(c, r_{1}\right)}\right]$ and $\left[b_{i j}^{\left(c, r_{2}\right)}\right]$ for $c_{1}=c_{2}=c$ is defined as $\left[a_{i j}^{\left(c, r_{1}\right)}\right] \sqcup_{c}\left[b_{i j}^{\left(c, r_{2}\right)}\right]=\left[d_{i j}\right] \in I S F M_{t \times v}$ where $d_{i j}=\max \left\{a_{i j}^{\left(c, r_{1}\right)}, b_{i j}^{\left(c, r_{2}\right)}\right\}$ for all $i$ and $j$;

b) The row-union ( $r$-union) of inverse fuzzy soft matrices $\left[a_{i j}^{\left(c_{1}, r\right)}\right]$ and $\left[b_{i j}^{\left(c_{2}, r\right)}\right]$ for $r_{1}=r_{2}=r$ is defined as $\left[a_{i j}^{\left(c_{1}, r\right)}\right] \sqcup_{r}\left[b_{i j}^{\left(c_{2}, r\right)}\right]=\left[d_{i j}\right] \in I S F M_{t \times v}$ where $d_{i j}=\max \left\{a_{i j}^{\left(c_{1}, r\right)}, b_{i j}^{\left(c_{2}, r\right)}\right\}$ for all $i$ and $j$; c) The union of inverse fuzzy soft matrices $\left[a_{i j}^{(c, r)}\right]$ and $\left[b_{i j}^{(c, r)}\right]$ for $c_{1}=c_{2}=c$ and $r_{1}=r_{2}=r$ is defined as $\left[a_{i j}^{(c, r)}\right] \sqcup\left[b_{i j}^{(c, r)}\right]=\left[d_{i j}\right] \in I S F M_{t \times v}$ where $d_{i j}=$ $\max \left\{a_{i j}^{(c, r)}, b_{i j}^{(c, r)}\right\}$ for all $i$ and $j$.

We can give the following example to show the authentic-life applications of $c$-operations.

Example 5. Assume that a bridegroom wants to compare the three brides from North India and South India to get married under the parameter set $X_{1}=\left\{x_{1}^{1}, x_{2}^{1}, x_{3}^{1}, x_{4}^{1}\right\}$, where $x_{1}^{1}$ represents $x_{1}^{1}$-Good character, $x_{2}^{1}$-Beauty, $x_{3}^{1}$-Finance awareness, and $x_{4}^{1}$ Excellent culture. Suppose that the brides of North India and South India are $O_{1}=\left\{o_{1}^{1}, o_{2}^{1}, o_{3}^{1}\right\}$ and $O_{2}=$ $\left\{o_{1}^{2}, o_{2}^{2}, o_{3}^{2}\right\}$, respectively. Then, it is seen that $O_{1} \cap O_{2}=$ $\emptyset$. According to the data, if the bridegroom constructs the following inverse fuzzy soft matrices:

$$
\begin{aligned}
& {\left[a_{i j}^{(1,1)}\right]=\left[\begin{array}{ccc}
0.6 & 0.5 & 0.4 \\
0.2 & 1 & 0.3 \\
0.8 & 0.1 & 0.9 \\
0 & 0.7 & 0.6
\end{array}\right],} \\
& {\left[b_{i k}^{(1,2)}\right]=\left[\begin{array}{ccc}
0.7 & 0.8 & 0.6 \\
0.3 & 1 & 0.5 \\
0.9 & 0.5 & 1 \\
1 & 0.8 & 0.7
\end{array}\right],}
\end{aligned}
$$

then he asserts that the South Indian brides are better than North Indian brides in all parameters, since $\left[a_{i j}^{(1,1)}\right] \sqsubseteq_{c}\left[b_{i k}^{(1,2)}\right]$.

If the bridegroom creates the following inverse fuzzy soft matrices:

$$
\begin{aligned}
& {\left[a_{i j}^{(1,1)}\right]=\left[\begin{array}{ccc}
0.6 & 0.8 & 0.9 \\
0.5 & 1 & 0.7 \\
1 & 0.7 & 0.4 \\
0.6 & 0.4 & 1
\end{array}\right],} \\
& {\left[b_{i k}^{(1,2)}\right]=\left[\begin{array}{ccc}
0.7 & 0.5 & 0.6 \\
0.4 & 1 & 0.6 \\
0.8 & 0.2 & 1 \\
1 & 0.7 & 1
\end{array}\right],}
\end{aligned}
$$

then he has the c-intersection of inverse fuzzy soft matrices $\left[a_{i j}^{(1,1)}\right]$ and $\left[b_{i k}^{(1,2)}\right]$ as follows:

$$
\left[a_{i j}^{(1,1)}\right] \Pi_{c}\left[b_{i k}^{(1,2)}\right]=\left[\begin{array}{ccc}
0.6 & 0.5 & 0.6 \\
0.4 & 1 & 0.6 \\
0.8 & 0.2 & 0.4 \\
0.6 & 0.4 & 1
\end{array}\right] .
$$

Thus, the bridegroom asserts that there is a girl from both North India and South India who is beautiful and has an excellent culture.

Moreover, many problems can be resolved by using the $r$-operations of inverse fuzzy soft matrices in authentic-life. 
Definition 11. Let $\left[a_{m j}^{\left(c_{1}, r\right)}\right] \in I S M_{t_{1} \times v}$ and $\left[b_{n j}^{\left(c_{2}, r\right)}\right] \in$ $I S M_{t_{2} \times v}$ :

a) The And row-product (And $r$-product) of inverse fuzzy soft matrices $\left[a_{m j}^{\left(c_{1}, r\right)}\right]$ and $\left[b_{n j}^{\left(c_{2}, r\right)}\right]$ is defined as $\left[a_{m j}^{\left(c_{1}, r\right)}\right] \wedge_{r}\left[b_{n j}^{\left(c_{2}, r\right)}\right]=\left[g_{s j}\right] \in I S M_{t_{1} t_{2} \times v}$, where:

$$
g_{s j}=\min \left\{a_{m j}^{\left(c_{1}, r\right)}, b_{n j}^{\left(c_{2}, r\right)}\right\}
$$

for $s=(m-1) t_{2}+n$.

b) The Or row-product (Or $r$-product) of inverse fuzzy soft matrices $\left[a_{m j}^{\left(c_{1}, r\right)}\right]$ and $\left[b_{n j}^{\left(c_{2}, r\right)}\right]$ is defined as $\left[a_{m j}^{\left(c_{1}, r\right)}\right] \vee_{r}\left[b_{n j}^{\left(c_{2}, r\right)}\right]=\left[h_{s j}\right] \in I S M_{t_{1} t_{2} \times v}$, where:

$$
h_{s j}=\max \left\{a_{m j}^{\left(c_{1}, r\right)}, b_{n j}^{\left(c_{2}, r\right)}\right\}
$$

for $s=(m-1) t_{2}+n$.

c) The exclusive Or row-product (exclusive Or $r$ product) of inverse fuzzy soft matrices $\left[a_{m j}^{\left(c_{1}, r\right)}\right]$ and $\left[b_{n j}^{\left(c_{2}, r\right)}\right]$ is defined as $\left[a_{m j}^{\left(c_{1}, r\right)}\right] \underline{\vee}_{r}\left[b_{n j}^{\left(c_{2}, r\right)}\right]=\left[h_{s j}\right] \in$ $I S M_{t_{1} t_{2} \times v}$, where:

$$
h_{s j}= \begin{cases}\max \left\{a_{m j}^{\left(c_{1}, r\right)}, b_{n j}^{\left(c_{2}, r\right)}\right\}, & \text { if } a_{m j}^{\left(c_{1}, r\right)} \neq b_{n j}^{\left(c_{2}, r\right)} \\ 0, & \text { if } a_{m j}^{\left(c_{1}, r\right)}=b_{n j}^{\left(c_{2}, r\right)}\end{cases}
$$

for $s=(m-1) t_{2}+n$.

Proposition 1. Let $\left[a_{m j}^{\left(c_{1}, r\right)}\right] \in \operatorname{IFSM}_{t_{1} \times v},\left[b_{n j}^{\left(c_{2}, r\right)}\right] \in$ $\operatorname{IFSM}_{t_{2} \times v}$ and $\left[c_{p j}^{\left(c_{3}, r\right)}\right] \in \operatorname{IFSM}_{t_{3} \times v}$. Also, $[1]_{1 \times v}$ represents the $1 \times v$ inverse fuzzy soft matrix with all components 1 and $[0]_{1 \times v}$ represents the $1 \times v$ inverse fuzzy soft matrix with all components 0 .

(i) $\left[a_{m j}^{\left(c_{1}, r\right)}\right] \wedge_{r}[1]_{1 \times v}=[1]_{1 \times v} \wedge_{r}\left[a_{m j}^{\left(c_{1}, r\right)}\right]=\left[a_{m j}^{\left(c_{1}, r\right)}\right]$;

(ii) $\left[a_{m j}^{\left(c_{1}, r\right)}\right] \vee_{r}[0]_{1 \times v}=[0]_{1 \times v} \vee_{r}\left[a_{m j}^{\left(c_{1}, r\right)}\right]=\left[a_{m j}^{\left(c_{1}, r\right)}\right]$;

(iii) $\left[a_{m j}^{\left(c_{1}, r\right)}\right] \underline{\vee}_{r}[0]_{1 \times v}=[0]_{1 \times v} \underline{\vee}_{r}\left[a_{m j}^{\left(c_{1}, r\right)}\right]=\left[a_{m j}^{\left(c_{1}, r\right)}\right]$;

(iv) $\left(\left[a_{m j}^{\left(c_{1}, r\right)}\right] *\left[b_{n j}^{\left(c_{2}, r\right)}\right]\right) *\left[c_{p j}^{\left(c_{3}, r\right)}\right]=\left[a_{m j}^{\left(c_{1}, r\right)}\right] *\left(\left[b_{n j}^{\left(c_{2}, r\right)}\right] *\right.$ $\left.\left[c_{p j}^{\left(c_{3}, r\right)}\right]\right)$ for each $* \in\left\{\wedge_{r}, \vee_{r}\right\}$.

Remark that it is usually $\left[a_{m j}^{\left(c_{1}, r\right)}\right] *\left[b_{n j}^{\left(c_{2}, r\right)}\right] \neq\left[b_{n j}^{\left(c_{2}, r\right)}\right] *$ $\left[a_{m j}^{\left(c_{1}, r\right)}\right]$ for each $* \in\left\{\wedge_{r}, \vee_{r}, \underline{\vee}_{r}\right\}$.

Proof. The proofs of (i)-(iii) are obvious in Definition 13. Therefore, they are omitted. Now, we achieve the proof of (iv) for the Or $r$-product $\mathrm{V}_{r}$.

Let $\left[a_{m j}^{\left(c_{1}, r\right)}\right] \in I F S M_{t_{1} \times v},\left[b_{n j}^{\left(c_{2}, r\right)}\right] \in I F S M_{t_{2} \times v}$ and $\left[c_{p j}^{\left(c_{3}, r\right)}\right] \in I F S M_{t_{3} \times v}$. By Definition $13(\mathrm{~b})$, we can write $\left[a_{m j}^{\left(c_{1}, r\right)}\right] \vee_{r}\left[b_{n j}^{\left(c_{2}, r\right)}\right]=\left[e_{q j}^{\left(\left\langle c_{1}, c_{2}\right\rangle, r\right)}\right] \in I F S M_{t_{1} t_{2} \times v}$ where $e_{q j}^{\left(\left\langle c_{1}, c_{2}\right\rangle, r\right)}=\max \left\{a_{m j}^{\left(c_{1}, r\right)}, b_{n j}^{\left(c_{2}, r\right)}\right\}$ for $q=(m-$
1) $t_{2}+n$. Besides, we have $\left[e_{q j}^{\left(\left\langle c_{1}, c_{2}\right\rangle, r\right)}\right] \vee_{r}\left[c_{p j}^{\left(c_{3}, r\right)}\right]=$ $\left[f_{s j}^{\left(\left\langle c_{1}, c_{2}, c_{3}\right\rangle, r\right)}\right] \in \operatorname{IFSM}_{t_{1} t_{2} t_{3} \times v}$ where:

$$
\begin{aligned}
f_{s j}^{\left(\left\langle c_{1}, c_{2}, c_{3}\right\rangle, r\right)} & =\max \left\{e_{q j}^{\left(\left\langle c_{1}, c_{2}\right\rangle, r\right)}, c_{p j}^{\left(c_{3}, r\right)}\right\} \\
& =\max \left\{\max \left\{a_{m j}^{\left(c_{1}, r\right)}, b_{n j}^{\left(c_{2}, r\right)}\right\}, c_{p j}^{\left(c_{3}, r\right)}\right\} \\
& =\max \left\{a_{m j}^{\left(c_{1}, r\right)}, b_{n j}^{\left(c_{2}, r\right)}, c_{p j}^{\left(c_{3}, r\right)}\right\}
\end{aligned}
$$

for

$$
\begin{aligned}
s & =(q-1) t_{3}+p=\left((m-1) t_{2}+n-1\right) t_{3}+p \\
& =(m-1) t_{2} t_{3}+(n-1) t_{3}+p .
\end{aligned}
$$

Similarly, we can write:

$$
\left[b_{n j}^{\left(c_{2}, r\right)}\right] \vee_{r}\left[c_{p j}^{\left(c_{3}, r\right)}\right]=\left[g_{q^{\prime} j}^{\left(\left\langle c_{2}, c_{3}\right\rangle, r\right)}\right] \in I F S M_{t_{2} t_{3} \times v}
$$

where $g_{q^{\prime} j}^{\left(\left\langle c_{2}, c_{3}\right\rangle, r\right)}=\max \left\{b_{n j}^{\left(c_{2}, r\right)}, c_{p j}^{\left(c_{3}, r\right)}\right\}$ for $q^{\prime}=(n-$ 1) $t_{3}+p$. Besides, we have:

$$
\left[a_{m j}^{\left(c_{1}, r\right)}\right] \vee_{r}\left[g_{q^{\prime} j}^{\left(\left\langle c_{2}, c_{3}\right\rangle, r\right)}\right]=\left[h_{s^{\prime} j}^{\left(\left\langle c_{1}, c_{2}, c_{3}\right\rangle, r\right)}\right] \in I F S M_{t_{1} t_{2} t_{3} \times v}
$$

where:

$$
\begin{aligned}
h_{s^{\prime} j}^{\left(\left\langle c_{1}, c_{2}, c_{3}\right\rangle, r\right)} & =\max \left\{a_{m j}^{\left(c_{1}, r\right)}, g_{q^{\prime} j}^{\left(\left\langle c_{2}, c_{3}\right\rangle, r\right)}\right\} \\
& =\max \left\{a_{m j}^{\left(c_{1}, r\right)}, \max \left\{b_{n j}^{\left(c_{2}, r\right)}, c_{p j}^{\left(c_{3}, r\right)}\right\}\right\} \\
& =\max \left\{a_{m j}^{\left(c_{1}, r\right)}, b_{n j}^{\left(c_{2}, r\right)}, c_{p j}^{\left(c_{3}, r\right)}\right\},
\end{aligned}
$$

for

$$
s^{\prime}=(m-1) t_{2} t_{3}+q^{\prime}=(m-1) t_{2} t_{3}+(n-1) t_{3}+p .
$$

By Eqs. (9), (10), (11), and (12), it is obtained that $\left[f_{s j}^{\left(\left\langle c_{1}, c_{2}, c_{3}\right\rangle, r\right)}\right]=\left[h_{s^{\prime} j}^{\left(\left\langle c_{1}, c_{2}, c_{3}\right\rangle, r\right)}\right]$. This completes the proof for the Or $r$-product $\vee_{r}$.

It can be proven in a similar way for the And $r$ product $\wedge_{r}$.

Note: If $\left(\left[a_{m j}^{\left(c_{1}, r\right)}\right],\left[b_{n j}^{\left(c_{2}, r\right)}\right]\right)$, and $\left[c_{p j}^{\left(c_{3}, r\right)}\right]$ are the inverse fuzzy soft matrices such that:

$$
a_{m j}^{\left(c_{1}, r\right)}, b_{n j}^{\left(c_{2}, r\right)}, c_{p j}^{\left(c_{3}, r\right)} \in\{0,1\},
$$

for all $1 \leq m \leq t_{1}, 1 \leq n \leq t_{2}, 1 \leq p \leq t_{3}, 1 \leq j \leq v$, then:

$$
\begin{aligned}
\left(\left[a_{m j}^{\left(c_{1}, r\right)}\right] \underline{\vee}_{r}\left[b_{n j}^{\left(c_{2}, r\right)}\right]\right) \underline{\vee}_{r}\left[c_{p j}^{\left(c_{3}, r\right)}\right]= \\
{\left[a_{m j}^{\left(c_{1}, r\right)}\right] \underline{\vee}_{r}\left(\left[b_{n j}^{\left(c_{2}, r\right)}\right] \underline{\vee}_{r}\left[c_{p j}^{\left(c_{3}, r\right)}\right]\right) . }
\end{aligned}
$$

Otherwise, generally:

$$
\begin{aligned}
\left(\left[a_{m j}^{\left(c_{1}, r\right)}\right] \underline{\vee}_{r}\left[b_{n j}^{\left(c_{2}, r\right)}\right]\right) \underline{\vee}_{r}\left[c_{p j}^{\left(c_{3}, r\right)}\right] \neq \\
{\left[a_{m j}^{\left(c_{1}, r\right)}\right] \underline{\vee}_{r}\left(\left[b_{n j}^{\left(c_{2}, r\right)}\right] \underline{\vee}_{r}\left[c_{p j}^{\left(c_{3}, r\right)}\right]\right) . }
\end{aligned}
$$

For this situation, we consider the following example. 
Example 6. We take the inverse fuzzy soft matrices $\left[a_{m j}^{(1,1)}\right] \in \operatorname{IFSM}_{2 \times 2},\left[b_{n j}^{(2,1)}\right] \in \mathrm{IFSM}_{2 \times 1}$ and $\left[c_{p j}^{(3,1)}\right] \in$ IFSM $_{2 \times 2}$ as follows:

$$
\begin{array}{ll}
{\left[a_{m j}^{(1,1)}\right.} & =\left[\begin{array}{cc}
0.4 & 0.2 \\
0.5 & 1
\end{array}\right], \quad\left[b_{n j}^{(2,1)}\right]=\left[\begin{array}{l}
0.5 \\
0.4
\end{array}\right], \\
{\left[c_{p j}^{(3,1)}\right.} & =\left[\begin{array}{cc}
0.5 & 0.7 \\
0.1 & 0
\end{array}\right] .
\end{array}
$$

Then, we have:

$$
\begin{aligned}
& \left.\left[a_{m j}^{\left(c_{1}, r\right)}\right] \underline{\vee}_{r}\left[b_{n j}^{\left(c_{2}, r\right)}\right]\right) \underline{\vee}_{r}\left[c_{p j}^{\left(c_{3}, r\right)}\right]=\left[\begin{array}{cc}
0 & 1 \\
0 & 0.5
\end{array}\right], \\
& {\left[a_{m j}^{\left(c_{1}, r\right)}\right] \underline{\vee}_{r}\left(\left[b_{n j}^{\left(c_{2}, r\right)}\right] \underline{\vee}_{r}\left[c_{p j}^{\left(c_{3}, r\right)}\right]=\left[\begin{array}{cc}
0.4 & 1 \\
0 & 0.5
\end{array}\right] .\right.}
\end{aligned}
$$

Thus, we have $\left(\left[a_{m j}^{\left(c_{1}, r\right)}\right] \underline{\vee}_{r}\left[b_{n j}^{\left(c_{2}, r\right)}\right]\right) \underline{\vee}_{r}\left[c_{p j}^{\left(c_{3}, r\right)}\right] \neq$ $\left[a_{m j}^{\left(c_{1}, r\right)}\right] \underline{\vee}_{r}\left(\left[b_{n j}^{\left(c_{2}, r\right)}\right] \underline{\vee}_{r}\left[c_{p j}^{\left(c_{3}, r\right)}\right]\right)$.

Theorem 1. According to each of the operations And $r$-product $\left(\wedge_{r}\right)$ and $\operatorname{Or}$ r-product $\left(\vee_{r}\right), \mathrm{IFSM}_{t \times v}$ is a monoid.

Proof. The proof is obvious by Proposition 1 .

Definition 12. Let $\left[a_{i j}^{\left(c, r_{1}\right)}\right] \in I S M_{t \times v_{1}}$ and $\left[b_{i k}^{\left(c, r_{2}\right)}\right] \in$ $I S M_{t \times v_{2}}$ :

a) The And column-product (And $c$-product) of inverse fuzzy soft matrices $\left[a_{i j}^{\left(c, r_{1}\right)}\right]$ and $\left[b_{i k}^{\left(c, r_{2}\right)}\right]$ is defined as $\left[a_{i j}^{\left(c, r_{1}\right)}\right] \wedge_{c}\left[b_{i k}^{\left(c, r_{2}\right)}\right]=\left[g_{i l}\right] \in I S M_{t \times v_{1} v_{2}}$, where:

$$
g_{i l}=\min \left\{a_{i j}^{\left(c, r_{1}\right)}, b_{i k}^{\left(c, r_{2}\right)}\right\},
$$

for $l=(j-1) v_{2}+k$.

b) The Or column-product (Or $c$-product) of inverse fuzzy soft matrices $\left[a_{i j}^{\left(c, r_{1}\right)}\right]$ and $\left[b_{i k}^{\left(c, r_{2}\right)}\right]$ is defined as $\left[a_{i j}^{\left(c, r_{1}\right)}\right] \vee_{c}\left[b_{i k}^{\left(c, r_{2}\right)}\right]=\left[h_{i l}\right] \in I S M_{t \times v_{1} v_{2}}$, where:

$$
h_{i l}=\max \left\{a_{i j}^{\left(c, r_{1}\right)}, b_{i k}^{\left(c, r_{2}\right)}\right\},
$$

for $l=(j-1) v_{2}+k$.

c) The exclusive Or column-product (exclusive Or $c$ product) of inverse fuzzy soft matrices $\left[a_{i j}^{\left(c, r_{1}\right)}\right]$ and $\left[b_{i k}^{\left(c, r_{2}\right)}\right]$ is defined as $\left[a_{i j}^{\left(c, r_{1}\right)}\right] \underline{\vee}_{c}\left[b_{i k}^{\left(c, r_{2}\right)}\right]=\left[h_{i l}\right] \in$ $I S M_{t \times v_{1} v_{2}}$, where:

$h_{i l}= \begin{cases}\max \left\{a_{i j}^{\left(c, r_{1}\right)}, b_{i k}^{\left(c, r_{2}\right)}\right\}, & \text { if } a_{i j}^{\left(c, r_{1}\right)} \neq b_{i k}^{\left(c, r_{2}\right)} \\ 0, & \text { if } a_{i j}^{\left(c, r_{1}\right)}=b_{i k}^{\left(c, r_{2}\right)}\end{cases}$

for $l=(j-1) v_{2}+k$.
Proposition 2. Let $\left[a_{i j}^{\left(c, r_{1}\right)}\right] \in \operatorname{IFSM}_{t \times v_{1}},\left[b_{i k}^{\left(c, r_{2}\right)}\right] \in$ $\operatorname{IFSM}_{t \times v_{2}}$ and $\left[c_{i l}^{\left(c, r_{3}\right)}\right] \in \operatorname{IFSM}_{t \times v_{3}}$. Also, [1 $]_{t \times 1}$ represents the $t \times 1$ inverse fuzzy soft matrix with all components 1 and $[0]_{t \times 1}$ represents the $t \times 1$ inverse fuzzy soft matrix with all components 0 .

(i) $\left[a_{i j}^{\left(c, r_{1}\right)}\right] \wedge_{c}[1]_{t \times 1}=[1]_{t \times 1} \wedge_{c}\left[a_{i j}^{\left(c, r_{1}\right)}\right]=\left[a_{i j}^{\left(c, r_{1}\right)}\right]$;

(ii) $\left[a_{i j}^{\left(c, r_{1}\right)}\right] \vee_{c}[0]_{t \times 1}=[0]_{t \times 1} \vee_{c}\left[a_{i j}^{\left(c, r_{1}\right)}\right]=\left[a_{i j}^{\left(c, r_{1}\right)}\right]$;

iii) $\left[a_{i j}^{\left(c, r_{1}\right)}\right] \underline{\vee}_{c}[0]_{t \times 1}=[0]_{t \times 1} \underline{\vee}_{c}\left[a_{i j}^{\left(c, r_{1}\right)}\right]=\left[a_{i j}^{\left(c, r_{1}\right)}\right]$;

iv) $\left(\left[a_{i j}^{\left(c, r_{1}\right)}\right] *\left[b_{i k}^{\left(c, r_{2}\right)}\right]\right) *\left[c_{i l}^{\left(c, r_{3}\right)}\right]=\left[a_{i j}^{\left(c, r_{1}\right)}\right] *\left(\left[b_{i k}^{\left(c, r_{2}\right)}\right] *\right.$ $\left.\left[c_{i l}^{\left(c, r_{3}\right)}\right]\right)$ for each $* \in\left\{\wedge_{c}, \vee_{c}\right\}$.

Remark that it is usually $\left[a_{i j}^{\left(c, r_{1}\right)}\right] *\left[b_{i k}^{\left(c, r_{2}\right)}\right] \neq$ $\left[b_{i k}^{\left(c, r_{2}\right)}\right] *\left[a_{i j}^{\left(c, r_{1}\right)}\right]$ for each $* \in\left\{\wedge_{c}, \vee_{c}, \underline{\vee}_{c}\right\}$.

Proof. The proof is similar to that of Proposition 2.

Note: If $\left(\left[a_{i j}^{\left(c, r_{1}\right)}\right],\left[b_{i k}^{\left(c, r_{2}\right)}\right]\right)$ and $\left[c_{i l}^{\left(c, r_{3}\right)}\right]$ are the inverse fuzzy soft matrices such that $a_{i j}^{\left(c, r_{1}\right)}, b_{i k}^{\left(c, r_{2}\right)}, c_{i l}^{\left(c, r_{3}\right)} \in$ $\{0,1\}$ for all $1 \leq i \leq t, 1 \leq j \leq v_{1}, 1 \leq k \leq v_{2}$, $1 \leq l \leq v_{3}$, then:

$$
\begin{aligned}
\left(\left[a_{i j}^{\left(c, r_{1}\right)}\right] \underline{\vee}_{c}\left[b_{i k}^{\left(c, r_{2}\right)}\right]\right) \underline{\vee}_{c}\left[c_{i l}^{\left(c, r_{3}\right)}\right]= \\
\\
{\left[a_{i j}^{\left(c, r_{1}\right)}\right] \underline{\vee}_{c}\left(\left[b_{i k}^{\left(c, r_{2}\right)}\right] \underline{\vee}_{c}\left[c_{i l}^{\left(c, r_{3}\right)}\right]\right) . }
\end{aligned}
$$

Otherwise, generally:

$$
\begin{aligned}
\left(\left[a_{i j}^{\left(c, r_{1}\right)}\right] \underline{\vee}_{c}\left[b_{i k}^{\left(c, r_{2}\right)}\right]\right) \underline{\vee}_{c}\left[c_{i l}^{\left(c, r_{3}\right)}\right] \neq \\
\\
{\left[a_{i j}^{\left(c, r_{1}\right)}\right] \underline{\vee}_{c}\left(\left[b_{i k}^{\left(c, r_{2}\right)}\right] \underline{\vee}_{c}\left[c_{i l}^{\left(c, r_{3}\right)}\right]\right) . }
\end{aligned}
$$

This can be exemplified in a similar way to the idea for Example 6.

Theorem 2. According to each of the operations And c-product $\left(\wedge_{c}\right)$ and Or c-product $\left(\vee_{c}\right), \operatorname{IFSM}_{t \times v}$ is a monoid.

Proof. The proof is obvious by Proposition 2 .

Example 7. Let us consider the inverse fuzzy soft matrices $\left[a_{i j}^{(1,1)}\right],\left[b_{i j}^{(1,1)}\right],\left[b_{i k}^{(1,2)}\right] \in \operatorname{IFSM}_{2 \times 3},\left[a_{m j}^{(2,1)}\right] \in$ $\mathrm{IFSM}_{3 \times 3}$ and $\left[b_{i l}^{(1,3)}\right] \in \mathrm{IFSM}_{2 \times 2}$ given in Example 3. Then, we obtain:

$$
\begin{aligned}
& {\left[a_{i j}^{(1,1)}\right] \wedge_{r}\left[b_{i j}^{(1,1)}\right]=\left[\begin{array}{lll}
0.4 & 0.9 & 0.3 \\
0.5 & 0.7 & 0.4 \\
0.4 & 0.9 & 0.3 \\
0.6 & 0.7 & 0.5
\end{array}\right],} \\
& {\left[a_{i j}^{(1,1)}\right] \wedge_{c}\left[b_{i l}^{(1,3)}\right]} \\
& =\left[\begin{array}{llllllll}
0.4 & 0.9 & 0.3 & 0.2 & 0.4 & 0.9 & 0.3 & 0.2 \\
0.4 & 0.9 & 0.3 & 0.2 & 0.4 & 0.9 & 0.3 & 0.2
\end{array}\right],
\end{aligned}
$$




$$
\begin{aligned}
& {\left[b_{i j}^{(1,1)}\right] \vee_{r}\left[a_{m j}^{(2,1)}\right]=\left[\begin{array}{lll}
0.6 & 0.9 & 0.3 \\
0.6 & 0.8 & 0.5 \\
0.4 & 0.9 & 0.3 \\
0.6 & 0.9 & 0.5 \\
0.4 & 0.9 & 0.7 \\
0.6 & 0.7 & 0.7
\end{array}\right],} \\
& {\left[b_{i j}^{(1,1)}\right] \underline{\vee}_{c}\left[b_{i l}^{(1,2)}\right]} \\
& =\left[\begin{array}{ccccccccc}
0 & 0.8 & 0.4 & 0.9 & 0.9 & 0.9 & 0.4 & 0.8 & 0 \\
0 & 0 & 0.6 & 0.7 & 0.7 & 0.7 & 0.5 & 0.6 & 0
\end{array}\right] .
\end{aligned}
$$

Definition 13. Let $\left[a_{m j}^{\left(c_{1}, r\right)}\right] \in I S M_{t_{1} \times v}$ and $\left[b_{n j}^{\left(c_{2}, r\right)}\right] \in$ $I S M_{t_{2} \times v}$ :

a) The difference row-product (difference $r$-product) of inverse fuzzy soft matrices $\left[a_{m j}^{\left(c_{1}, r\right)}\right]$ and $\left[b_{n j}^{\left(c_{2}, r\right)}\right]$ is defined as $\left[a_{m j}^{\left(c_{1}, r\right)}\right] \frown_{r}\left[b_{n j}^{\left(c_{2}, r\right)}\right]=\left[f_{s j}\right] \in \operatorname{ISM}_{t_{1} t_{2} \times v}$, where:

$$
f_{s j}=\left\{\begin{array}{lll}
a_{m j}^{\left(c_{1}, r\right)}-b_{n j}^{\left(c_{2}, r\right)}, & \text { if } & a_{m j}^{\left(c_{1}, r\right)} \geq b_{n j}^{\left(c_{2}, r\right)} \\
0, & \text { if } & a_{m j}^{\left(c_{1}, r\right)}<b_{n j}^{\left(c_{2}, r\right)}
\end{array}\right.
$$

for $s=(m-1) t_{2}+n$.

b) The symmetric difference row-product (symmetric difference $r$-product) of inverse fuzzy soft matrices $\left[a_{m j}^{\left(c_{1}, r\right)}\right]$ and $\left[b_{n j}^{\left(c_{2}, r\right)}\right]$ is defined as $\left[a_{m j}^{\left(c_{1}, r\right)}\right] \asymp_{r}$ $\left[b_{n j}^{\left(c_{2}, r\right)}\right]=\left[f_{s j}\right] \in I S M_{t_{1} t_{2} \times v}$, where:

$$
f_{s j}=\left|a_{m j}^{\left(c_{1}, r\right)}-b_{n j}^{\left(c_{2}, r\right)}\right|,
$$

for $s=(m-1) t_{2}+n$.

Proposition 3. Let $\left[a_{m j}^{\left(c_{1}, r\right)}\right] \in \operatorname{IFSM}_{t_{1} \times v}$ and $\left[b_{n j}^{\left(c_{2}, r\right)}\right] \in$ $\mathrm{IFSM}_{t_{2} \times v}$. Also, $[1]_{t_{1} \times v}$ represents the $t_{1} \times v$ inverse fuzzy soft matrix with all components 1 and $[0]_{t_{1} \times v}$ represents the $t_{1} \times v$ inverse fuzzy soft matrix with all components 0 .

(i) $\left[a_{m j}^{\left(c_{1}, r\right)}\right] *\left[a_{m j}^{\left(c_{1}, r\right)}\right]=[0]_{t_{1} \times v}$ for each $* \in\left\{\frown_{r}, \asymp_{r}\right\}$;

(ii) $[1]_{1 \times v} *\left[a_{m j}^{\left(c_{1}, r\right)}\right]=\left[a_{m j}^{\left(c_{1}, r\right)}\right]^{\circ}$ for each $* \in\left\{\frown_{r}, \asymp_{r}\right\}$;

(iii) $\left[a_{m j}^{\left(c_{1}, r\right)}\right] *[0]_{1 \times v}=\left[a_{m j}^{\left(c_{1}, r\right)}\right]$ for each $* \in\left\{\frown_{r}, \asymp_{r}\right\}$.

Note that usually $\left[a_{m j}^{\left(c_{1}, r\right)}\right] *\left[b_{n j}^{\left(c_{2}, r\right)}\right] \neq\left[b_{n j}^{\left(c_{2}, r\right)}\right] *\left[a_{m j}^{\left(c_{1}, r\right)}\right]$ for each $* \in\left\{\frown_{r}, \asymp_{r}\right\}$.

Proof. It is clear by Definition 13 .

Definition 14. Let $\left[a_{i j}^{\left(c, r_{1}\right)}\right] \in I S M_{t \times v_{1}}$ and $\left[b_{i k}^{\left(c, r_{2}\right)}\right] \in$ $I S M_{t \times v_{2}}$ :

a) The difference column-product (difference $c$ product) of inverse fuzzy soft matrices $\left[a_{i j}^{\left(c, r_{1}\right)}\right]$ and $\left[b_{i k}^{\left(c, r_{2}\right)}\right]$ is defined as $\left[a_{i j}^{\left(c, r_{1}\right)}\right] \frown_{c}\left[b_{i k}^{\left(c, r_{2}\right)}\right]=\left[f_{i l}\right] \in$ $I S M_{t \times v_{1} v_{2}}$, where:

$$
f_{i l}=\left\{\begin{array}{lll}
a_{i j}^{\left(c, r_{1}\right)}-b_{i k}^{\left(c, r_{2}\right)}, & \text { if } & a_{i j}^{\left(c, r_{1}\right)} \geq b_{i k}^{\left(c, r_{2}\right)} \\
0, & \text { if } & a_{i j}^{\left(c,, r_{1}\right)}<b_{i k}^{\left(c, r_{2}\right)}
\end{array}\right.
$$

for $l=(j-1) v_{2}+k$.

b) The symmetric difference column-product (symmetric difference $c$-product) of inverse fuzzy soft matrices $\left[a_{i j}^{\left(c, r_{1}\right)}\right]$ and $\left[b_{i k}^{\left(c, r_{2}\right)}\right]$ is defined as $\left[a_{i j}^{\left(c, r_{1}\right)}\right] \asymp_{c}\left[b_{i k}^{\left(c, r_{2}\right)}\right]=\left[f_{i l}\right] \in I S M_{t \times v_{1} v_{2}}$, where:

$$
f_{i l}=\left|a_{i j}^{\left(c, r_{1}\right)}-b_{i k}^{\left(c, r_{2}\right)}\right|,
$$

for $l=(j-1) v_{2}+k$.

Proposition 4. Let $\left[a_{i j}^{\left(c, r_{1}\right)}\right] \in \operatorname{IFSM}_{t \times v_{1}}$ and $\left[b_{i k}^{\left(c, r_{2}\right)}\right] \in$ $\mathrm{IFSM}_{t \times v_{2}}$. Also, $[1]_{t \times v_{1}}$ represents the $t \times v_{1}$ inverse fuzzy soft matrix with all components 1 and $[0]_{t \times v_{1}}$ represents the $t \times v_{1}$ inverse fuzzy soft matrix with all components 0 .

(i) $\left[a_{i j}^{\left(c, r_{1}\right)}\right] *\left[a_{i j}^{\left(c, r_{1}\right)}\right]=[0]_{t \times v_{1}}$ for each $* \in\left\{\frown_{c}, \asymp_{c}\right\}$;

(ii) $[1]_{t \times 1} *\left[a_{i j}^{\left(c, r_{1}\right)}\right]=\left[a_{i j}^{\left(c, r_{1}\right)}\right]^{\circ}$ for each $* \in\left\{\frown_{c}, \asymp_{c}\right\}$;

(iii) $\left[a_{i j}^{\left(c, r_{1}\right)}\right] *[0]_{t \times 1}=\left[a_{i j}^{\left(c, r_{1}\right)}\right]$ for each $* \in\left\{\frown_{c}, \asymp_{c}\right\}$.

Note that usually $\left[a_{i j}^{\left(c, r_{1}\right)}\right] *\left[b_{i k}^{\left(c, r_{2}\right)}\right] \neq\left[b_{i k}^{\left(c, r_{2}\right)}\right] *\left[a_{i j}^{\left(c, r_{1}\right)}\right]$ for each $* \in\left\{\frown_{c}, \smile_{c}\right\}$.

Proof. It is clear by Definition 14 .

Example 8. Let us consider the inverse fuzzy soft matrices $\left[a_{i j}^{(1,1)}\right],\left[b_{i j}^{(1,1)}\right] \in \mathrm{IFSM}_{2 \times 3},\left[a_{m j}^{(2,1)}\right] \in \mathrm{IFSM}_{3 \times 3}$ and $\left[b_{i l}^{(1,3)}\right] \in \mathrm{IFSM}_{2 \times 2}$ given in Example 3. Then, we obtain:

$$
\left[a_{i j}^{(1,1)}\right] \neg_{c}\left[b_{i l}^{(1,3)}\right]=\left[\begin{array}{cccccc}
0.3 & 0.2 & 0.7 & 0.6 & 0.2 & 0.1 \\
0 & 0.5 & 0.3 & 0.9 & 0 & 0.6
\end{array}\right],
$$

and

$$
\left[b_{i j}^{(1,1)}\right] \asymp_{r}\left[a_{m j}^{(2,1)}\right]=\left[\begin{array}{ccc}
0.2 & 0.1 & 0.2 \\
0.1 & 0 & 0.1 \\
0 & 0.5 & 0.4 \\
0 & 0.1 & 0.4 \\
0.3 & 0.2 & 0.3 \\
0.2 & 0.3 & 0.2
\end{array}\right] .
$$

Definition 15. Let $\left[a_{m j}^{\left(c_{1}, r\right)}\right] \in I S M_{t_{1} \times v}$ and $\left[b_{n j}^{\left(c_{2}, r\right)}\right] \in$ $I S M_{t_{2} \times v}$ :

a) The right-If row-product (right-If $r$-product) of inverse fuzzy soft matrices $\left[a_{m j}^{\left(c_{1}, r\right)}\right]$ and $\left[b_{n j}^{\left(c_{2}, r\right)}\right]$ is defined as $\left[a_{m j}^{\left(c_{1}, r\right)}\right] \Rightarrow_{r}\left[b_{n j}^{\left(c_{2}, r\right)}\right]=\left[f_{s j}\right] \in I S M_{t_{1} t_{2} \times v}$, where:

$$
f_{s j}= \begin{cases}1, & \text { if } a_{m j}^{\left(c_{1}, r\right)} \leq b_{n j}^{\left(c_{2}, r\right)} \\ 0, & \text { otherwise }\end{cases}
$$

for $s=(m-1) t_{2}+n$. 
b) The left-If row-product (left-If $r$-product) of inverse fuzzy soft matrices $\left[a_{m j}^{\left(c_{1}, r\right)}\right]$ and $\left[b_{n j}^{\left(c_{2}, r\right)}\right]$ is defined as $\left[a_{m j}^{\left(c_{1}, r\right)}\right] \Leftarrow_{r}\left[b_{n j}^{\left(c_{2}, r\right)}\right]=\left[g_{s j}\right] \in I S M_{t_{1} t_{2} \times v}$, where:

$$
g_{s j}= \begin{cases}1, & \text { if } a_{m j}^{\left(c_{1}, r\right)} \geq b_{n j}^{\left(c_{2}, r\right)} \\ 0, & \text { otherwise }\end{cases}
$$

for $s=(m-1) t_{2}+n$.

c) The Iff row-product (Iff $r$-product) of inverse fuzzy soft matrices $\left[a_{m j}^{\left(c_{1}, r\right)}\right]$ and $\left[b_{n j}^{\left(c_{2}, r\right)}\right]$ is defined as $\left[a_{m j}^{\left(c_{1}, r\right)}\right] \Leftrightarrow_{r}\left[b_{n j}^{\left(c_{2}, r\right)}\right]=\left[h_{s j}\right] \in I S M_{t_{1} t_{2} \times v}$, where:

$$
h_{s j}= \begin{cases}1, & \text { if } a_{m j}^{\left(c_{1}, r\right)}=b_{n j}^{\left(c_{2}, r\right)} \\ 0, & \text { otherwise }\end{cases}
$$

for $s=(m-1) t_{2}+n$.

Proposition 5. Let $\left[a_{m j}^{\left(c_{1}, r\right)}\right] \in \operatorname{IFSM}_{t_{1} \times v}$ and $\left[b_{n j}^{\left(c_{2}, r\right)}\right] \in$ $\operatorname{IFSM}_{t_{2} \times v}$. Then, $\left(\left[a_{m j}^{\left(c_{1}, r\right)}\right] \Rightarrow_{r}\left[b_{n j}^{\left(c_{2}, r\right)}\right]\right) \sqcap\left(\left[b_{n j}^{\left(c_{2}, r\right)}\right] \Leftarrow_{r}\right.$ $\left.\left[a_{m j}^{\left(c_{1}, r\right)}\right]\right)=\left[a_{m j}^{\left(c_{1}, r\right)}\right] \Leftrightarrow_{r}\left[b_{n j}^{\left(c_{2}, r\right)}\right]$.

Proof. We consider that $\left[f_{s j}\right]=\left[a_{m j}^{\left(c_{1}, r\right)}\right] \Rightarrow_{r}\left[b_{n j}^{\left(c_{2}, r\right)}\right]$ and $\left[g_{s j}\right]=\left[b_{n j}^{\left(c_{2}, r\right)}\right] \Leftarrow_{r}\left[a_{m j}^{\left(c_{1}, r\right)}\right]$. Then, by Definition 15 (a) and (b), we have for all $m, n$, and $j$ :

$$
f_{s j}=\left\{\begin{array}{lll}
1, & \text { if } & a_{m j}^{\left(c_{1}, r\right)} \leq b_{n j}^{\left(c_{2}, r\right)} \\
0, & \text { if } & a_{m j}^{\left(c_{1}, r\right)}>b_{n j}^{\left(c_{2}, r\right)}
\end{array}\right.
$$

and

$$
g_{s j}=\left\{\begin{array}{lll}
1, & \text { if } \quad a_{m j}^{\left(c_{1}, r\right)} \geq b_{n j}^{\left(c_{2}, r\right)} \\
0, & \text { if } \quad a_{m j}^{\left(c_{1}, r\right)}<b_{n j}^{\left(c_{2}, r\right)}
\end{array}\right.
$$

Thus, we can write $\left[e_{s j}\right]=\left(\left[a_{m j}^{\left(c_{1}, r\right)}\right] \Rightarrow_{r}\left[b_{n j}^{\left(c_{2}, r\right)}\right]\right) \sqcap$ $\left(\left[a_{m j}^{\left(c_{1}, r\right)}\right] \Leftarrow_{r}\left[b_{n j}^{\left(c_{2}, r\right)}\right]\right)$ such that for all $m, n$, and $j$ :

$$
\begin{gathered}
e_{s j}=\left\{\begin{array}{lll}
\min \{1,1\}, & \text { if } & a_{m j}^{\left(c_{1}, r\right)}=b_{n j}^{\left(c_{2}, r\right)} \\
\min \{1,0\}, & \text { if } & a_{m j}^{\left(c_{1}, r\right)}<b_{n j}^{\left(c_{2}, r\right)} \\
\min \{0,1\}, & \text { if } & a_{m j}^{\left(c_{1}, r\right)}>b_{n j}^{\left(c_{2}, r\right)}
\end{array}\right. \\
=\left\{\begin{array}{lll}
1, & \text { if } \quad a_{m j}^{\left(c_{1}, r\right)}=b_{n j}^{\left(c_{2}, r\right)} \\
0, & \text { if } \quad a_{m j}^{\left(c_{1}, r\right)} \neq b_{n j}^{\left(c_{2}, r\right)}
\end{array}\right.
\end{gathered}
$$

From Definition 15(c), it can be easily seen that $\left(\left[a_{m j}^{\left(c_{1}, r\right)}\right] \Rightarrow_{r}\left[b_{n j}^{\left(c_{2}, r\right)}\right]\right) \sqcap\left(\left[b_{n j}^{\left(c_{2}, r\right)}\right] \quad \Leftarrow_{r}\left[a_{m j}^{\left(c_{1}, r\right)}\right]\right)=$ $\left[a_{m j}^{\left(c_{1}, r\right)}\right] \Leftrightarrow_{r}\left[b_{n j}^{\left(c_{2}, r\right)}\right]$.

Definition 16. Let $\left[a_{i j}^{\left(c, r_{1}\right)}\right] \in I S M_{t \times v_{1}}$ and $\left[b_{i k}^{\left(c, r_{2}\right)}\right] \in$ $I S M_{t \times v_{2}}$ :

a) The right-If column-product (right-If $c$-product) of inverse fuzzy soft matrices $\left[a_{i j}^{\left(c, r_{1}\right)}\right]$ and $\left[b_{i k}^{\left(c, r_{2}\right)}\right]$ is defined as $\left[a_{i j}^{\left(c, r_{1}\right)}\right] \Rightarrow_{c}\left[b_{i k}^{\left(c, r_{2}\right)}\right]=\left[f_{i l}\right] \in I S M_{t \times v_{1} v_{2}}$, where:

$$
f_{i l}= \begin{cases}1, & \text { if } a_{i j}^{\left(c, r_{1}\right)} \leq b_{i k}^{\left(c, r_{2}\right)} \\ 0, & \text { otherwise }\end{cases}
$$

for $l=(j-1) v_{2}+k$.

b) The left-If column-product (left-If $c$-product) of inverse fuzzy soft matrices $\left[a_{i j}^{\left(c, r_{1}\right)}\right]$ and $\left[b_{i k}^{\left(c, r_{2}\right)}\right]$ is defined as $\left[a_{i j}^{\left(c, r_{1}\right)}\right] \Leftarrow_{c}\left[b_{i k}^{\left(c, r_{2}\right)}\right]=\left[g_{i l}\right] \in I S M_{t \times v_{1} v_{2}}$, where:

$$
g_{i l}= \begin{cases}1, & \text { if } a_{i j}^{\left(c, r_{1}\right)} \geq b_{i k}^{\left(c, r_{2}\right)} \\ 0, & \text { otherwise }\end{cases}
$$

for $l=(j-1) v_{2}+k$.

c) The Iff column-product (Iff $c$-product) of inverse fuzzy soft matrices $\left[a_{i j}^{\left(c, r_{1}\right)}\right]$ and $\left[b_{i k}^{\left(c, r_{2}\right)}\right]$ is defined as $\left[a_{i j}^{\left(c, r_{1}\right)}\right] \Leftrightarrow_{c}\left[b_{i k}^{\left(c, r_{2}\right)}\right]=\left[h_{i l}\right] \in I S M_{t \times v_{1} v_{2}}$, where:

$$
h_{i l}= \begin{cases}1, & \text { if } a_{i j}^{\left(c, r_{1}\right)}=b_{i k}^{\left(c, r_{2}\right)} \\ 0, & \text { otherwise }\end{cases}
$$

for $l=(j-1) v_{2}+k$.

Proposition 6. Let $\left[a_{i j}^{\left(c, r_{1}\right)}\right] \in \operatorname{IFSM}_{t \times v_{1}}$ and $\left[b_{i k}^{\left(c, r_{2}\right)}\right] \in$ $\operatorname{IFSM}_{t \times v_{2}}$. Then, $\left(\left[a_{i j}^{\left(c, r_{1}\right)}\right] \Rightarrow_{c}\left[b_{i k}^{\left(c, r_{2}\right)}\right]\right) \sqcap\left(\left[b_{i k}^{\left(c, r_{2}\right)}\right] \Leftarrow_{c}\right.$ $\left.\left[a_{i j}^{\left(c, r_{1}\right)}\right]\right)=\left[a_{i j}^{\left(c, r_{1}\right)}\right] \Leftrightarrow_{c}\left[b_{i k}^{\left(c, r_{2}\right)}\right]$.

Proof. This proof is similar to the proof for Proposition 5 , so it is omitted.

Example 9. Let us consider the inverse fuzzy soft matrices $\left[b_{i j}^{(1,1)}\right],\left[b_{i k}^{(1,2)}\right] \in \operatorname{IFSM}_{2 \times 3},\left[a_{m j}^{(2,1)}\right] \in \mathrm{IFSM}_{3 \times 3}$, and $\left[b_{i l}^{(1,3)}\right] \in \mathrm{IFSM}_{2 \times 2}$ given in Example 3. Then, we have the following inverse fuzzy soft matrices:

$$
\left[a_{m j}^{(2,1)}\right] \Leftarrow_{r}\left[b_{i j}^{(1,1)}\right]=\left[\begin{array}{ccc}
1 & 0 & 0 \\
1 & 1 & 0 \\
0 & 1 & 0 \\
0 & 1 & 0 \\
1 & 0 & 1 \\
0 & 0 & 1
\end{array}\right],
$$$$
\left[b_{i j}^{(1,1)}\right] \Leftarrow_{r}\left[a_{m j}^{(2,1)}\right]=\left[\begin{array}{lll}
0 & 1 & 1 \\
1 & 1 & 1 \\
1 & 1 & 0 \\
1 & 0 & 1 \\
1 & 0 & 1 \\
1 & 1 & 0
\end{array}\right],
$$

$$
\left[b_{i k}^{(1,2)}\right] \Rightarrow_{c}\left[b_{i l}^{(1,3)}\right]=\left[\begin{array}{llllll}
0 & 0 & 0 & 0 & 0 & 1 \\
1 & 0 & 1 & 0 & 1 & 0
\end{array}\right] \text {, }
$$




$$
\begin{gathered}
{\left[b_{i l}^{(1,3)}\right] \Rightarrow_{c}\left[b_{i k}^{(1,2)}\right]=\left[\begin{array}{llllll}
1 & 1 & 1 & 1 & 1 & 1 \\
0 & 0 & 0 & 1 & 1 & 1
\end{array}\right],} \\
{\left[b_{i k}^{(1,2)}\right] \Leftarrow_{c}\left[b_{i l}^{(1,3)}\right]=\left[\begin{array}{llllll}
1 & 1 & 1 & 1 & 1 & 1 \\
0 & 1 & 0 & 1 & 0 & 1
\end{array}\right],}
\end{gathered}
$$

and

$$
\left[b_{i k}^{(1,2)}\right] \Leftrightarrow_{c}\left[b_{i l}^{(1,3)}\right]=\left[\begin{array}{llllll}
0 & 0 & 0 & 0 & 0 & 1 \\
0 & 0 & 0 & 0 & 0 & 0
\end{array}\right] .
$$

Thus, it is seen that $\left(\left[b_{i k}^{(1,2)}\right] \Rightarrow_{c}\left[b_{i l}^{(1,3)}\right]\right) \sqcap\left(\left[b_{i k}^{(1,2)}\right] \Leftarrow_{c}\right.$ $\left.\left[b_{i l}^{(1,3)}\right]\right)=\left[b_{i k}^{(1,2)}\right] \Leftrightarrow b_{c}\left[b_{i l}^{(1,3)}\right]$. Also, by the above example, it is obvious that $\left(\left[b_{i k}^{(1,2)}\right] \Rightarrow_{c}\left[b_{i l}^{(1,3)}\right]\right) \neq$ $\left[b_{i l}^{(1,3)}\right] \Rightarrow_{c}\left[b_{i k}^{(1,2)}\right]$.

\section{The IFSSPDM method}

In this section, the And sum-product, Or sum-product, mean sum-product, and aggregate mean sum-product decision inverse fuzzy soft matrices are introduced. Later on, we DEVELOP an MCGDM algorithm called Inverse Fuzzy Soft Sum-Product Decision Making (IFSSPDM) algorithm (Algorithm 1) to find an optimum universal object set $O$ according to the multiplediscrete parameter sets. To apprehend Algorithm 1 more clearly, a flow diagram (Figure 1) is given. The implementation of the elaborated algorithm is a decision to appoint a new vice-chancellor at a university in India.

\subsection{The algorithm of IFSSPDM and its application}

Definition 17. Let $\left[a_{m j}^{\left(c_{1}, r\right)}\right] \in I F S M_{t_{1} \times v}$ and $\left[b_{n j}^{\left(c_{2}, r\right)}\right] \in$ $\operatorname{IFSM}_{t_{2} \times v}$. If $\left[a_{m j}^{\left(c_{1}, r\right)}\right] \wedge_{r}\left[b_{n j}^{\left(c_{2}, r\right)}\right]=\left[g_{s j}\right] \in I F S M_{t_{1} t_{2} \times v}$, then the matrix $\left[y_{1 j}\right]$ is called an And sum-product decision inverse fuzzy soft matrix, where for each $j=$ $1,2, \cdots, v$ :

$$
y_{1 j}=\frac{1}{t_{1}\left(t_{2}+1\right)} \sum_{\ell=1}^{t_{1}} \alpha_{\ell j}, \quad\left(0 \leq y_{1 j} \leq 1\right),
$$

such that for each $\ell=1,2, \cdots, t_{1}$ :

$$
\alpha_{\ell j}=\sum_{s=(\ell-1) t_{2}+1}^{\ell t_{2}} g_{s j}+\prod_{s=(\ell-1) t_{2}+1}^{\ell t_{2}} g_{s j} .
$$

Definition 18. Let $\left[a_{m j}^{\left(c_{1}, r\right)}\right] \in I F S M_{t_{1} \times v}$ and $\left[b_{n j}^{\left(c_{2}, r\right)}\right] \in$ $I F S M_{t_{2} \times v}$. If $\left[a_{m j}^{\left(c_{1}, r\right)}\right] \vee_{r}\left[b_{n j}^{\left(c_{2}, r\right)}\right]=\left[h_{s^{\prime} j}\right] \in I F S M_{t_{1} t_{2} \times v}$, then the matrix $\left[z_{1 j}\right]$ is called an Or sum-product decision inverse fuzzy soft matrix, where for each $j=$ $1,2, \cdots, v$ :

$$
z_{1 j}=\frac{1}{t_{1}\left(t_{2}+1\right)} \sum_{\ell=1}^{t_{1}} \gamma_{\ell j}, \quad\left(0 \leq z_{1 j} \leq 1\right) .
$$

such that for each $\ell=1,2, \cdots, t_{1}$ :

$$
\gamma_{\ell j}=\sum_{s^{\prime}=(\ell-1) t_{2}+1}^{\ell t_{2}} h_{s^{\prime} j}+\prod_{s^{\prime}=(\ell-1) t_{2}+1}^{\ell t_{2}} h_{s^{\prime} j} .
$$

Definition 19. Let $\left[y_{1 j}\right] \in I F S M_{1 \times v}$ and $\left[z_{1 j}\right] \in$ $I F S M_{1 \times v}$ be the And and Or product-sum decision inverse fuzzy soft matrices, respectively. Then, the matrix $\left[v_{1 j}\right]$ is called a mean sum-product decision inverse fuzzy soft matrix, where for each $j=1,2, \cdots, v$ :

$$
v_{1 j}=\frac{y_{1 j}+z_{1 j}}{2}, \quad\left(0 \leq v_{1 j} \leq 1\right)
$$

Definition 20. Let $\left[v_{1 j}^{1}\right],\left[v_{1 j}^{2}\right], \cdots,\left[v_{1 j}^{k}\right] \in I F S M_{1 \times v}$ be the mean product-sum decision inverse fuzzy soft matrices. Then, the matrix $\left[w_{1 j}\right]$ is called an aggregate mean sum-product decision inverse fuzzy soft matrix, where for each $j=1,2, \cdots, v$ :

$$
w_{1 j}=\frac{1}{k} \sum_{i=1}^{k} v_{1 j}^{i}, \quad\left(0 \leq w_{1 j} \leq 1\right) .
$$

Definition 21. Let $O_{1}=\left\{o_{1}^{1}, o_{2}^{1}, \cdots, o_{v}^{1}\right\}$ be a universal set. By using the aggregate mean sumproduct decision inverse fuzzy soft matrix $\left[w_{1 j}\right]$, we get the ranking order of objects as follows:

$$
o_{j_{1}}^{1}>o_{j_{2}}^{1}>\cdots>o_{j_{v}}^{1} \quad \text { if } \quad w_{1 j_{1}}>w_{1 j_{2}}>\cdots>w_{1 j_{n}} .
$$

Then, the optimum set of $O$ is obtained as follows:

$$
\begin{gathered}
\mathcal{S}_{\left[w_{1 j}\right]}^{o p t}\left(O_{1}\right)=\left\{o_{j}^{1}: o_{j}^{1} \in O_{1} \text { and } w_{1 j}>w_{1 j^{\prime}}\right. \\
\text { for } \left.\forall j^{\prime} \neq j\right\} .
\end{gathered}
$$

By using the decision inverse fuzzy soft matrices described above, the following adjustable algorithm emerges. This algorithm can be successfully applied to the MCGDM based on the four inverse fuzzy soft sets over a common universal set $O$.

\section{Algorithm 1.}

Step 1. Create the inverse fuzzy soft matrices (represented as $A=\left[a_{m j}^{\left(c_{1}, r\right)}\right], B=\left[b_{n j}^{\left(c_{2}, r\right)}\right], C=\left[c_{p j}^{\left(c_{3}, r\right)}\right]$, and $\left.D=\left[d_{q j}^{\left(c_{4}, r\right)}\right]\right)$ from the given four inverse fuzzy soft sets;

Step 2. Obtain the And $r$-products:

$$
\begin{array}{ll}
{\left[a_{m j}^{\left(c_{1}, r\right)}\right] \wedge_{r}\left[b_{n j}^{\left(c_{2}, r\right)}\right],} & {\left[a_{m j}^{\left(c_{1}, r\right)}\right] \wedge_{r}\left[c_{p j}^{\left(c_{3}, r\right)}\right],} \\
{\left[a_{m j}^{\left(c_{1}, r\right)}\right] \wedge_{r}\left[d_{q j}^{\left(c_{4}, r\right)}\right],} & {\left[b_{n j}^{\left(c_{2}, r\right)}\right] \wedge_{r}\left[c_{p j}^{\left(c_{3}, r\right)}\right],} \\
{\left[b_{n j}^{\left(c_{2}, r\right)}\right] \wedge_{r}\left[d_{q j}^{\left(c_{4}, r\right)}\right],} & {\left[c_{p j}^{\left(c_{3}, r\right)}\right] \wedge_{r}\left[d_{q j}^{\left(c_{4}, r\right)}\right] .}
\end{array}
$$

Step 3. Obtain the Or $r$-products: 


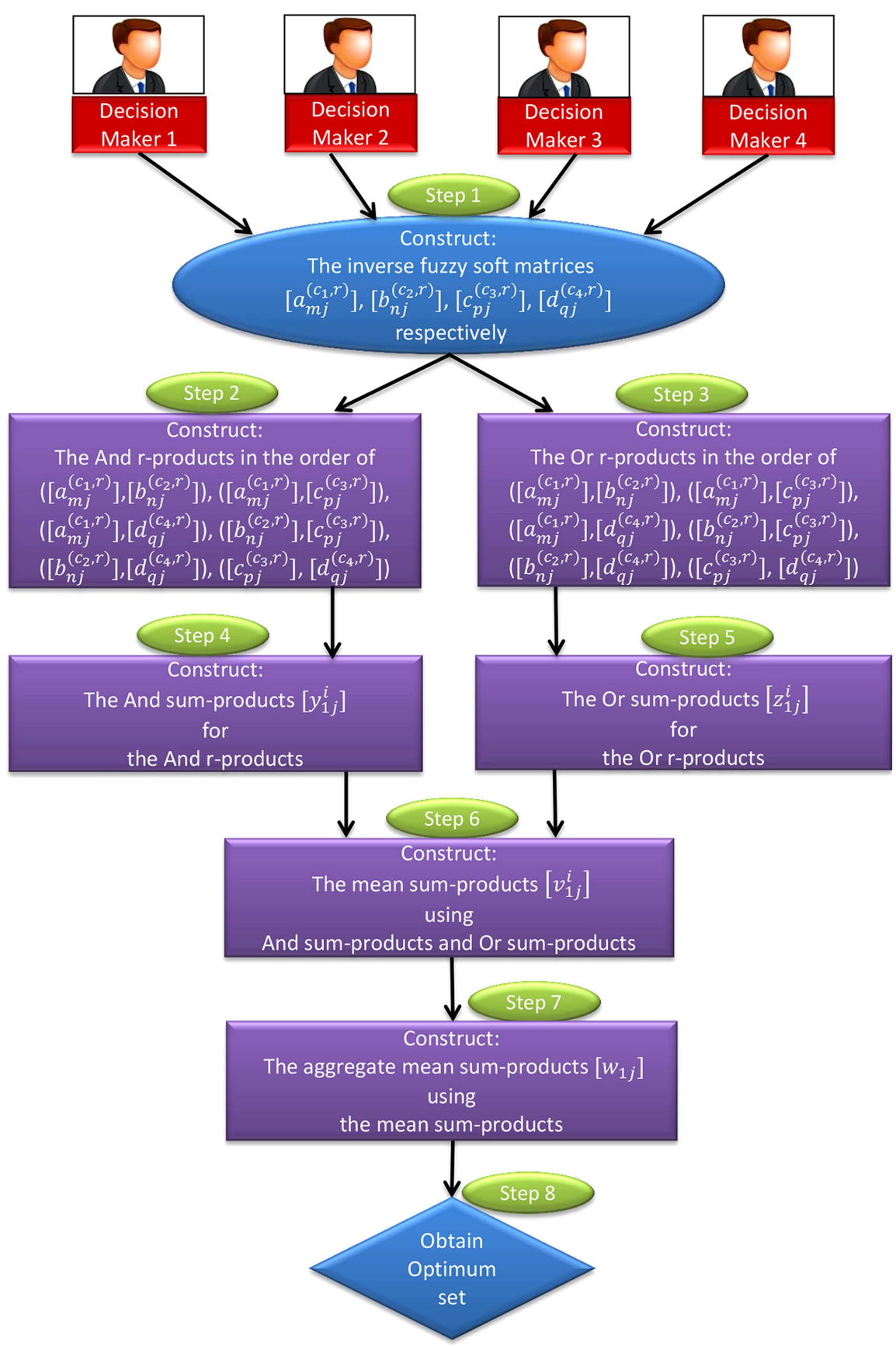

Figure 1. The flow diagram of Algorithm 1.

$$
\begin{array}{ll}
{\left[a_{m j}^{\left(c_{1}, r\right)}\right] \vee_{r}\left[b_{n j}^{\left(c_{2}, r\right)}\right],} & {\left[a_{m j}^{\left(c_{1}, r\right)}\right] \vee_{r}\left[c_{p j}^{\left(c_{3}, r\right)}\right],} \\
{\left[a_{m j}^{\left(c_{1}, r\right)}\right] \vee_{r}\left[d_{q j}^{\left(c_{4}, r\right)}\right],} & {\left[b_{n j}^{\left(c_{2}, r\right)}\right] \vee_{r}\left[c_{p j}^{\left(c_{3}, r\right)}\right],} \\
{\left[b_{n j}^{\left(c_{2}, r\right)}\right] \vee_{r}\left[d_{q j}^{\left(c_{4}, r\right)}\right],} & {\left[c_{p j}^{\left(c_{3}, r\right)}\right] \vee_{r}\left[d_{q j}^{\left(c_{4}, r\right)}\right] .}
\end{array}
$$

Step 4. Find the And sum-product decision inverse fuzzy soft matrices $\left[y_{1 j}^{i}\right] i \in\{1,2, \cdots, 6\}$ as follows:

$$
\left[y_{1 j}^{1}\right] \text { for }\left[a_{m j}^{\left(c_{1}, r\right)}\right] \wedge_{r}\left[b_{n j}^{\left(c_{2}, r\right)}\right]
$$

$\left[y_{1 j}^{2}\right]$ for $\left[a_{m j}^{\left(c_{1}, r\right)}\right] \wedge_{r}\left[c_{p j}^{\left(c_{3}, r\right)}\right]$

$\left[y_{1 j}^{3}\right]$ for $\left[a_{m j}^{\left(c_{1}, r\right)}\right] \wedge_{r}\left[d_{q j}^{\left(c_{4}, r\right)}\right]$,

$\left[y_{1 j}^{4}\right]$ for $\left[b_{n j}^{\left(c_{2}, r\right)}\right] \wedge_{r}\left[c_{p j}^{\left(c_{3}, r\right)}\right]$,

$\left[y_{1 j}^{5}\right]$ for $\left[b_{n j}^{\left(c_{2}, r\right)}\right] \wedge_{r}\left[d_{q j}^{\left(c_{4}, r\right)}\right]$,

$\left[y_{1 j}^{6}\right]$ for $\left[c_{p j}^{\left(c_{3}, r\right)}\right] \wedge_{r}\left[d_{q j}^{\left(c_{4}, r\right)}\right]$.

Step 5. Find the Or sum-product decision inverse 
fuzzy soft matrices $\left[y_{1 j}^{i}\right]$ for all $i \in\{1,2, \cdots, 6\}$ as follows:

$$
\begin{aligned}
& {\left[z_{1 j}^{1}\right] \text { for }\left[a_{m j}^{\left(c_{1}, r\right)}\right] \vee_{r}\left[b_{n j}^{\left(c_{2}, r\right)}\right],} \\
& {\left[z_{1 j}^{2}\right] \text { for }\left[a_{m j}^{\left(c_{1}, r\right)}\right] \vee_{r}\left[c_{p j}^{\left(c_{3}, r\right)}\right],} \\
& {\left[z_{1 j}^{3}\right] \text { for }\left[a_{m j}^{\left(c_{1}, r\right)}\right] \vee_{r}\left[d_{q j}^{\left(c_{4}, r\right)}\right],} \\
& {\left[z_{1 j}^{4}\right] \text { for }\left[b_{n j}^{\left(c_{2}, r\right)}\right] \vee_{r}\left[c_{p j}^{\left(c_{3}, r\right)}\right],} \\
& {\left[z_{1 j}^{5}\right] \text { for }\left[b_{n j}^{\left(c_{2}, r\right)}\right] \vee_{r}\left[d_{q j}^{\left(c_{4}, r\right)}\right],} \\
& {\left[z_{1 j}^{6}\right] \text { for }\left[c_{p j}^{\left(c_{3}, r\right)}\right] \vee_{r}\left[d_{q j}^{\left(c_{4}, r\right)}\right] .}
\end{aligned}
$$

Step 6. Find the mean sum-product decision inverse fuzzy soft matrices $\left[v_{1 j}^{i}\right]$ for all $i \in\{1,2, \cdots, 6\}$, where:

$$
v_{1 j}^{i}=\frac{y_{1 j}^{i}+z_{1 j}^{i}}{2},
$$

for each $i=1,2, \cdots, 6$.

Step 7. Find the aggregate mean sum-product decision inverse fuzzy soft matrix $\left[w_{1 j}\right]$, where

$$
w_{1 j}=\frac{1}{6} \sum_{i=1}^{6} v_{1 j}^{i},
$$

for each $j=1,2, \cdots, 6$.

Step 8. Obtain an optimum set $\mathcal{S}_{\left[w_{1 j}\right]}^{o p t}\left(O_{1}\right)$ considering the aggregate mean sum-product decision inverse fuzzy soft matrix.

Now let us visualize Algorithm 1 with an extraordinary example of MCGDM.

Example 10. Suppose that the Government of Tamilnadu in India decides to appoint a new vicechancellor at Anna University, Chennai. For that purpose, the state governor forms a committee of expert members under the head of a retired justice. The committee identifies four types of parameters which the next vice-chancellor can possess. The first parameter set, named "Personalities," is $X_{1}=\left\{x_{1}^{1}, x_{2}^{1}, x_{3}^{1}\right\}$ with $x_{1}^{1}$ persons of the highest level of competence, $x_{2}^{1}$ persons of the highest level of integrity, and $x_{3}^{1}$ persons of the highest level of morality. The second parameter set, named "Essential Criteria," is $X_{2}=$ $\left\{x_{1}^{2}, x_{2}^{2}, x_{3}^{2}, x_{4}^{2}, x_{5}^{2}, x_{6}^{2}\right\}$ with $x_{1}^{2}$ people having a terminal degree from a reputed institution, $x_{2}^{2}$ people having outstanding academic record with a minimum of 15 years of experience in teaching and research, $x_{3}^{2}$ people having as many years of experience as a principal/dean/director in a developed institution, $x_{4}^{2}$ persons having participated in/organized national and international seminars/conferences/workshops, $x_{5}^{2}$ people with publications of quality research papers/books, and $x_{6}^{2}$ persons having demonstrated academic leadership in higher education. The third parameter set, named "Desirable Attributes," is $X_{3}=\left\{x_{1}^{3}, x_{2}^{3}, x_{3}^{3}, x_{4}^{3}, x_{5}^{3}\right\}$ with $x_{1}^{3}$ having experience of guiding doctoral scholars and executing funded research projects, $x_{2}^{3}$ having experience as a visiting professor in foreign countries, $x_{3}^{3}$ involved in youth and women development activities, $x_{4}^{3}$ involved in students assessments and counseling, and $x_{5}^{3}$ having any awards/patent rights/other recognition. The fourth parameter set, named "Administrative Skills," is $X_{4}=\left\{x_{1}^{4}, x_{2}^{4}, x_{3}^{4}, x_{4}^{4}, x_{5}^{4}\right\}$, where $x_{1}^{4}$ have the vision to prove the leadership qualities; $x_{2}^{4}$ have a mission to develop the institution into world standard; $x_{3}^{4}$ are quick and perfect decision makers; $x_{4}^{4}$ have the skills to motivate, encourage, and inspire others; and $x_{5}^{4}$ are coordinated with government departments and statutory bodies at the state and national levels. The above selection parameters were partially framed by E. Balagurusamy, The former Tamilnadu Planning Commission's Member (Education), which was published in the newspaper "The Hindu" on 03.04.2012 (ref:https://bit.ly/2Mx9weh). The committee received about 170 application forms from all over the country after advertising through news channels and others. They eliminated 140 applications by three rounds of basic screening test and ranked the remaining candidates based on their qualifications. Following the ranking process, they short-listed 6 candidates $O_{1}=$ $\left\{o_{1}^{1}, o_{2}^{1}, o_{3}^{1}, o_{4}^{1}, o_{5}^{1}, o_{6}^{1}\right\}$ and prepared the following questionnaire by giving marks between 0 to 1 for the final selection. It was very difficult and time consuming for the committee to select the most suitable final candidate. This is while the IFSSP MCGDM algorithm (Algorithm 1) could help them achieve their task quickly and wisely.

To use the IFSSP MCGDM algorithm, we must construct the inverse fuzzy soft matrices from the questionnaire (Table 1). Therefore, we first generate the inverse fuzzy soft sets as shown in Box I. Now we can use Algorithm 1.

Step 1. The inverse fuzzy soft matrices of the inverse fuzzy soft sets $\tilde{\Psi}_{X_{1}}^{O_{1}}, \tilde{\Psi}_{X_{2}}^{O_{1}}, \tilde{\Psi}_{X_{3}}^{O_{1}}$, and $\tilde{\Psi}_{X_{4}}^{O_{1}}$ are respectively:

$$
\begin{aligned}
& {\left[a_{m j}^{(1,1)}\right]=\left[\begin{array}{cccccc}
0.5 & 0.3 & 0.5 & 0 & 0 & 0.2 \\
0 & 0.4 & 0.6 & 0.7 & 0.5 & 0 \\
0.7 & 0.8 & 0.8 & 0 & 0.8 & 0.9
\end{array}\right],} \\
& {\left[b_{n j}^{(2,1)}\right]=\left[\begin{array}{cccccc}
1 & 0.7 & 1 & 1 & 0.1 & 0 \\
0 & 1 & 0.5 & 0.5 & 0.5 & 0.5 \\
0.3 & 0.3 & 0.8 & 0.2 & 0.3 & 0.2 \\
0.7 & 0 & 0.9 & 0.6 & 0.7 & 0.7 \\
1 & 0.2 & 1 & 0 & 0.1 & 1 \\
0.1 & 0.1 & 0.1 & 0.2 & 0 & 0.8
\end{array}\right]}
\end{aligned}
$$


Table 1. Evaluation questionnaire of candidates by the committee to select the vice-chancellor.

\begin{tabular}{|c|c|c|c|c|c|c|c|c|c|c|c|c|c|c|c|c|c|c|c|c|}
\hline & \multicolumn{3}{|c|}{ Personalities } & \multicolumn{6}{|c|}{ Essential criteria } & \multicolumn{5}{|c|}{ Desirable attributes } & \multicolumn{5}{|c|}{ Administrative skills } \\
\hline & & $x_{1}^{1}$ & $x_{2}^{1}$ & $x_{3}^{1}$ & $x_{1}^{2}$ & $x_{2}^{2}$ & $x_{3}^{2}$ & $x_{4}^{2}$ & $x_{5}^{2}$ & $x_{6}^{2}$ & $x_{1}^{3}$ & $x_{2}^{3}$ & $x_{3}^{3}$ & $x_{4}^{3}$ & $x_{5}^{3}$ & $x_{1}^{4}$ & $x_{2}^{4}$ & $x_{3}^{4}$ & $\boldsymbol{x}_{4}^{4}$ & $x_{5}^{4}$ \\
\hline \multirow{6}{*}{ Candidates } & $o_{1}^{1}$ & 0.5 & 0 & 0.7 & 1 & 0 & 0.3 & 0.7 & 1 & 0.1 & 0 & 0.6 & 1 & 0.3 & 0.5 & 0 & 0.3 & 1 & 0.6 & 0.6 \\
\hline & $o_{2}^{1}$ & 0.3 & 0.4 & 0.8 & 0.7 & 1 & 0.3 & 0 & 0.2 & 0.1 & 0.9 & 0.7 & 1 & 0 & 0.2 & 0.9 & 0.9 & 1 & 0.7 & 0.2 \\
\hline & $o_{3}^{1}$ & 0.5 & 0.6 & 0.8 & 1 & 0.5 & 0 & 0.9 & 1 & 0.1 & 0.1 & 0.3 & 0.7 & 0.3 & 0.4 & 0.1 & 0.8 & 0.7 & 0 & 0.4 \\
\hline & $o_{4}^{1}$ & 0 & 0.7 & 0 & 1 & 0.5 & 0.2 & 0.6 & 0 & 0.2 & 0.2 & 0.4 & 0.5 & 0.8 & 0.5 & 0.2 & 0.7 & 0.5 & 0.9 & 0.5 \\
\hline & $o_{5}^{1}$ & 0 & 0.5 & 0.8 & 0.1 & 0.5 & 0.3 & 0.7 & 0.1 & 0 & 0.1 & 0.3 & 0.7 & 0.3 & 0.4 & 0.1 & 0.5 & 0.6 & 0.5 & 0 \\
\hline & $o_{6}^{1}$ & 0.2 & 0 & 0.9 & 0 & 0.5 & 0.2 & 0.7 & 1 & 0.8 & 0.4 & 0.5 & 0.7 & 0 & 0.2 & 0.4 & 0.5 & 0.8 & 0.8 & 0.3 \\
\hline
\end{tabular}

$$
\tilde{\Psi}_{X_{1}}^{O_{1}}=\left\{\begin{array}{l}
\left(o_{1}^{1},\left\{\left(x_{1}^{1}, 0.5\right),\left(x_{2}^{1}, 0\right),\left(x_{3}^{1}, 0.7\right)\right\}\right) \\
\left(o_{2}^{1},\left\{\left(x_{1}^{1}, 0.3\right),\left(x_{2}^{1}, 0.4\right),\left(x_{3}^{1}, 0.8\right)\right\}\right) \\
\left(o_{3}^{1},\left\{\left(x_{1}^{1}, 0.5\right),\left(x_{2}^{1}, 0.6\right),\left(x_{3}^{1}, 0.8\right)\right\}\right), \\
\left(o_{4}^{1},\left\{\left(x_{1}^{1}, 0\right),\left(x_{2}^{1}, 0.7\right),\left(x_{3}^{1}, 0\right)\right\}\right) \\
\left(o_{5}^{1},\left\{\left(x_{1}^{1}, 0\right),\left(x_{2}^{1}, 0.5\right),\left(x_{3}^{1}, 0.8\right)\right\}\right) \\
\left(o_{6}^{1},\left\{\left(x_{1}^{1}, 0.2\right),\left(x_{2}^{1}, 0\right),\left(x_{3}^{1}, 0.9\right)\right\}\right)
\end{array}\right\}
$$$$
\tilde{\Psi}_{X_{2}}^{O_{1}}=\left\{\begin{array}{l}
\left(o_{1}^{1},\left\{\left(x_{1}^{2}, 1\right),\left(x_{2}^{2}, 0\right),\left(x_{3}^{2}, 0.3\right),\left(x_{4}^{2}, 0.7\right),\left(x_{5}^{2}, 1\right),\left(x_{6}^{2}, 0.1\right)\right\}\right), \\
\left(o_{2}^{1},\left\{\left(x_{1}^{2}, 0.7\right),\left(x_{2}^{2}, 1\right),\left(x_{3}^{2}, 0.3\right),\left(x_{4}^{2}, 0\right),\left(x_{5}^{2}, 0.2\right),\left(x_{6}^{2}, 0.1\right)\right\}\right), \\
\left(o_{3}^{1},\left\{\left(x_{1}^{2}, 1\right),\left(x_{2}^{2}, 0.5\right),\left(x_{3}^{2}, 0\right),\left(x_{4}^{2}, 0.9\right),\left(x_{5}^{2}, 1\right),\left(x_{6}^{2}, 0.1\right)\right\}\right), \\
\left(o_{4}^{1},\left\{\left(x_{1}^{2}, 1\right),\left(x_{2}^{2}, 0.5\right),\left(x_{3}^{2}, 0.2\right),\left(x_{4}^{2}, 0.6\right),\left(x_{5}^{2}, 0\right),\left(x_{6}^{2}, 0.2\right)\right\}\right), \\
\left(o_{5}^{1},\left\{\left(x_{1}^{2}, 0.1\right),\left(x_{2}^{2}, 0.5\right),\left(x_{3}^{2}, 0.3\right),\left(x_{4}^{2}, 0.7\right),\left(x_{5}^{2}, 0.1\right),\left(x_{6}^{2}, 0\right)\right\}\right), \\
\left(o_{6}^{1},\left\{\left(x_{1}^{2}, 0\right),\left(x_{2}^{2}, 0.5\right),\left(x_{3}^{2}, 0.2\right),\left(x_{4}^{2}, 0.7\right),\left(x_{5}^{2}, 1\right),\left(x_{6}^{2}, 0.8\right)\right\}\right)
\end{array}\right\},
$$$$
\tilde{\Psi}_{X_{3}}^{O_{1}}=\left\{\begin{array}{l}
\left(o_{1}^{1},\left\{\left(x_{1}^{3}, 0\right),\left(x_{2}^{3}, 0.6\right),\left(x_{3}^{3}, 1\right),\left(x_{4}^{3}, 0.3\right),\left(x_{5}^{3}, 0.5\right)\right\}\right), \\
\left(o_{2}^{1},\left\{\left(x_{1}^{3}, 0.9\right),\left(x_{2}^{3}, 0.7\right),\left(x_{3}^{3}, 1\right),\left(x_{4}^{3}, 0\right),\left(x_{5}^{3}, 0.2\right)\right\}\right), \\
\left(o_{3}^{1},\left\{\left(x_{1}^{3}, 0.1\right),\left(x_{2}^{3}, 0.3\right),\left(x_{3}^{3}, 0.7\right),\left(x_{4}^{3}, 0.3\right),\left(x_{5}^{3}, 0.4\right)\right\}\right), \\
\left(o_{4}^{1},\left\{\left(x_{1}^{3}, 0.2\right),\left(x_{2}^{3}, 0.4\right),\left(x_{3}^{3}, 0.5\right),\left(x_{4}^{3}, 0.8\right),\left(x_{5}^{3}, 0.5\right)\right\}\right), \\
\left(o_{5}^{1},\left\{\left(x_{1}^{3}, 0.1\right),\left(x_{2}^{3}, 0.3\right),\left(x_{3}^{3}, 0.7\right),\left(x_{4}^{3}, 0.3\right),\left(x_{5}^{3}, 0.4\right)\right\}\right), \\
\left(o_{6}^{1},\left\{\left(x_{1}^{3}, 0.4\right),\left(x_{2}^{3}, 0.5\right),\left(x_{3}^{3}, 0.7\right),\left(x_{4}^{3}, 0\right),\left(x_{5}^{3}, 0.2\right)\right\}\right)
\end{array}\right\},
$$$$
\tilde{\Psi}_{X_{4}}^{O_{1}}=\left\{\begin{array}{l}
\left(o_{1}^{1},\left\{\left(x_{1}^{4}, 0\right),\left(x_{2}^{4}, 0.3\right),\left(x_{3}^{4}, 1\right),\left(x_{4}^{4}, 0.6\right),\left(x_{5}^{4}, 0.6\right)\right\}\right), \\
\left(o_{2}^{1},\left\{\left(x_{1}^{4}, 0.9\right),\left(x_{2}^{4}, 0.9\right),\left(x_{3}^{4}, 1\right),\left(x_{4}^{4}, 0.7\right),\left(x_{5}^{4}, 0.2\right)\right\}\right), \\
\left(o_{3}^{1},\left\{\left(x_{1}^{4}, 0.1\right),\left(x_{2}^{4}, 0.8\right),\left(x_{3}^{4}, 0.7\right),\left(x_{4}^{4}, 0\right),\left(x_{5}^{4}, 0.4\right)\right\}\right), \\
\left(o_{4}^{1},\left\{\left(x_{1}^{4}, 0.2\right),\left(x_{2}^{4}, 0.7\right),\left(x_{3}^{4}, 0.5\right),\left(x_{4}^{4}, 0.9\right),\left(x_{5}^{4}, 0.5\right)\right\}\right), \\
\left(o_{5}^{1},\left\{\left(x_{1}^{4}, 0.1\right),\left(x_{2}^{4}, 0.5\right),\left(x_{3}^{4}, 0.6\right),\left(x_{4}^{4}, 0.5\right),\left(x_{5}^{4}, 0\right)\right\}\right), \\
\left(o_{6}^{1},\left\{\left(x_{1}^{4}, 0.4\right),\left(x_{2}^{4}, 0.5\right),\left(x_{3}^{4}, 0.8\right),\left(x_{4}^{4}, 0.8\right),\left(x_{5}^{4}, 0.3\right)\right\}\right)
\end{array}\right\} .
$$ 


$$
\begin{aligned}
{\left[c_{p j}^{(3,1)}\right] } & =\left[\begin{array}{cccccc}
0 & 0.9 & 0.1 & 0.2 & 0.1 & 0.4 \\
0.6 & 0.7 & 0.3 & 0.4 & 0.3 & 0.5 \\
1 & 1 & 0.7 & 0.5 & 0.7 & 0.7 \\
0.3 & 0 & 0.3 & 0.8 & 0.3 & 0 \\
0.5 & 0.2 & 0.4 & 0.5 & 0.4 & 0.2
\end{array}\right] \\
{\left[d_{q j}^{(4,1)}\right] } & =\left[\begin{array}{cccccc}
0 & 0.9 & 0.1 & 0.2 & 0.1 & 0.4 \\
0.3 & 0.9 & 0.8 & 0.7 & 0.5 & 0.5 \\
1 & 1 & 0.7 & 0.5 & 0.6 & 0.8 \\
0.6 & 0.7 & 0.5 & 0.9 & 0.5 & 0.8 \\
0.6 & 0.2 & 0.4 & 0.5 & 0 & 0.3
\end{array}\right]
\end{aligned}
$$

Step 2-3. Since the dimensions of these inverse soft matrices are large, we can obtain And-products and Or-products using the Scilab codes in Appendix. Hence, these steps are omitted;

Step 4. The And sum-product decision inverse soft matrices are found as follows:

$\left[y_{1 j}^{1}\right]=$

$\left[\begin{array}{llllll}0.2095 & 0.223 & 0.449 & 0.1047 & 0.1523 & 0.1952\end{array}\right]$,

$\left[y_{1 j}^{2}\right]=$

$\left[\begin{array}{llllll}0.2166 & 0.2777 & 0.2836 & 0.1285 & 0.1891 & 0.1444\end{array}\right]$,

$\left[y_{1 j}^{3}\right]=$

$\left[\begin{array}{llllll}0.2222 & 0.3654 & 0.3735 & 0.1458 & 0.1833 & 0.2132\end{array}\right]$,

$\left[y_{1 j}^{4}\right]=$

$\left[\begin{array}{llllll}0.2361 & 0.2055 & 0.2586 & 0.2427 & 0.1584 & 0.2166\end{array}\right]$

$\left[y_{1 j}^{5}\right]=$

$\left[\begin{array}{llllll}0.2444 & 0.2712 & 0.3486 & 0.2628 & 0.1416 & 0.32\end{array}\right]$,

$\left[y_{1 j}^{6}\right]=$

$\left[\begin{array}{llllll}0.2533 & 0.3854 & 0.2404 & 0.3319 & 0.18 & 0.2584\end{array}\right]$.

Step 5. The Or sum-product decision inverse soft matrices are found as follows:

$\left[z_{1 j}^{1}\right]=$

$\left[\begin{array}{llllll}0.5917 & 0.55 & 0.7523 & 0.4603 & 0.4754 & 0.6048\end{array}\right]$,

$\left[z_{1 j}^{2}\right]=$

$\left[\begin{array}{llllll}0.5341 & 0.639 & 0.5701 & 0.4791 & 0.4929 & 0.4942\end{array}\right]$,

$\left[z_{1 j}^{3}\right]=$

$\left[\begin{array}{llllll}0.5461 & 0.723 & 0.601 & 0.5321 & 0.4813 & 0.5981\end{array}\right]$,

$$
\begin{aligned}
& {\left[z_{1 j}^{4}\right]=} \\
& {\left[\begin{array}{llllll}
0.6576 & 0.6195 & 0.7212 & 0.5389 & 0.384 & 0.5706
\end{array}\right],} \\
& {\left[z_{1 j}^{5}\right]=} \\
& {\left[\begin{array}{llllll}
0.6662 & 0.7224 & 0.7499 & 0.5891 & 0.3839 & 0.6417
\end{array}\right],} \\
& {\left[z_{1 j}^{6}\right]=} \\
& {\left[\begin{array}{llllll}
0.605 & 0.7826 & 0.4871 & 0.5573 & 0.4106 & 0.5242
\end{array}\right] \text {. }}
\end{aligned}
$$

Step 6. The mean sum-product decision inverse soft matrices are found as follows:

$\left[v_{1 j}^{1}\right]=$

$\left[\begin{array}{llllll}0.4006 & 0.3865 & 0.6006 & 0.2825 & 0.3138 & 0.4\end{array}\right]$,

$\left[v_{1 j}^{2}\right]=$

$\left[\begin{array}{llllll}0.3753 & 0.4583 & 0.4268 & 0.3038 & 0.341 & 0.3193\end{array}\right]$,

$\left[v_{1 j}^{3}\right]=$

$\left[\begin{array}{llllll}0.3841 & 0.5442 & 0.4872 & 0.3389 & 0.3323 & 0.4056\end{array}\right]$,

$\left[v_{1 j}^{4}\right]=$

$\left[\begin{array}{llllll}0.4468 & 0.4125 & 0.4899 & 0.3908 & 0.2712 & 0.3936\end{array}\right]$,

$\left[v_{1 j}^{5}\right]=$

$\left[\begin{array}{llllll}0.4553 & 0.4968 & 0.5492 & 0.4259 & 0.2627 & 0.4808\end{array}\right]$,

$\left[v_{1 j}^{6}\right]=$

$\left[\begin{array}{llllll}0.4291 & 0.584 & 0.3637 & 0.4446 & 0.2953 & 0.3913\end{array}\right]$.

Step 7. The aggregate mean sum-product decision inverse soft matrix is found as:

$\left[w_{1 j}\right]=$

$\left[\begin{array}{llllll}0.4152 & 0.4803 & 0.4862 & 0.3644 & 0.3027 & 0.3984\end{array}\right]$.

Step 8. Then, we achieve the ranking order of objects as $o_{3}^{1} \succ o_{2}^{1} \succ o_{1}^{1} \succ o_{6}^{1} \succ o_{4}^{1} \succ o_{5}^{1}$. Thus, the optimum set of $O_{1}$ is $\mathcal{S}_{\left[w_{1 j}\right]}^{\mathrm{opt}}\left(O_{1}\right)=\left\{o_{3}^{1}\right\}$. Accordingly, we suggest the committee to choose candidate $o_{3}^{1}$, as the most suitable (optimum) candidate for the post of vice-chancellor as per their identified parameters.

\subsection{Advantages of the IFSSPDM method (Algorithm 1) in comparison}

A fuzzy soft set can be uniquely represented as an inverse fuzzy soft set and vice versa. Here, the outputs 
Table 2. Comparison of the IFSSPDM method (Algorithm 1) with other methods.

\begin{tabular}{|c|c|c|c|}
\hline Problem & Method & Ranking order & $\begin{array}{c}\text { Ranking order of } \\
\text { proposed Algorithm } 1\end{array}$ \\
\hline Example 6 in [8] & Çag̃man and Enginog̃lu [8] & $\left\{u_{1}\right\}$ & $u_{2} \succ u_{1} \succ u_{3} \succ u_{5} \succ u_{4}$ \\
\hline Example 5.6 in [9] & Atagün et al. [9] & $\left\{u_{1}, u_{3}\right\}$ & $u_{3} \succ u_{1} \succ u_{4} \succ u_{5} \succ u_{2}$ \\
\hline Example 5.1 in [28] & Çag̃man and Enginog̃lu [28] & $\left\{u_{1}\right\}$ & $u_{3} \succ u_{1} \succ u_{2} \succ u_{5} \succ u_{4}$ \\
\hline Example 4.4 in [31] & Inthumathi et al. [31] & $\left\{u_{1}\right\}$ & $u_{1} \succ u_{4} \succ u_{5} \succ u_{2} \succ u_{3}$ \\
\hline Example 6.4 in [13] & Kamac1 et al. [13] & $l_{1} \succ l_{3} \succ l_{4} \succ l_{2}$ & $l_{1} \succ l_{4} \succ l_{3} \succ l_{2}$ \\
\hline Case study in [18] & Das and Borgohain [18] & $\left\{c_{3}\right\}$ & $c_{2} \succ c_{3} \succ c_{1}$ \\
\hline $\begin{array}{l}\text { Problem in Section } 2 \\
\text { in [19] }\end{array}$ & Gogoi et al. [19] & $e \succ c \succ m \succ p \succ h$ & $e \succ m \succ c \succ p \succ h$ \\
\hline $\begin{array}{l}\text { Numerical Example } \\
\text { in }[27]\end{array}$ & $\begin{array}{l}\text { Sandhiya and } \\
\text { Selvakumari }[27]\end{array}$ & $p_{4} \succ p_{3} \succ p_{1} \succ p_{2}$ & $p_{4} \succ p_{3} \succ p_{1} \succ p_{2}$ \\
\hline $\begin{array}{l}\text { Problem in } \\
\text { Section } 5 \text { in [29] }\end{array}$ & Borah et al. [29] & $c_{4} \succ c_{3} \succ c_{5} \succ c_{2} \succ c_{1}$ & $c_{4} \succ c_{3} \succ c_{5} \succ c_{2} \succ c_{1}$ \\
\hline $\begin{array}{l}\text { Problem in } \\
\text { Section 4.1.1 in [30] }\end{array}$ & Razak and Mohamad [30] & $m_{6} \succ m_{5} \succ m_{1} \succ m_{2}=m_{4} \succ m_{3}=m_{7}$ & $m_{5} \succ m_{7} \succ m_{6} \succ m_{3} \succ m_{1} \succ m_{4} \succ m_{2}$ \\
\hline
\end{tabular}

Table 3. Advantages of the IFSSPDM method (Algorithm 1).

\begin{tabular}{|c|c|c|c|c|c|}
\hline Method/ Algorithm & $\begin{array}{c}\text { Number of } \\
\text { criteria } \\
\text { used }\end{array}$ & $\begin{array}{c}\text { Each } \\
\text { decision } \\
\text { maker } \\
\text { can have } \\
\text { different } \\
\text { criteria }\end{array}$ & $\begin{array}{c}\text { Calculation } \\
\text { strategic }\end{array}$ & $\begin{array}{l}\text { Possible to } \\
\text { increase } \\
\text { number of } \\
\text { criteria }\end{array}$ & $\begin{array}{c}\text { Number of } \\
\text { decision } \\
\text { makers }\end{array}$ \\
\hline Çag̃man and Enginog̃lu [8] & 4 & No & Manual & Yes $^{*}$ & 2 \\
\hline Atagün et al. [9] & 6 & Yes & Manual \& Scilab codes & Yes & 2 \\
\hline Çag̃man and Enginog̃lu [28] & 4 & No & Manual & Yes $^{*}$ & 2 \\
\hline Inthumathi et al. [31] & 4 & No & Manual & Yes $^{*}$ & 2 \\
\hline Kamacı et al. [13] & 24 & Yes & Manual \& Scilab codes & Yes & Finitely many \\
\hline Das and Borgohain [18] & 3 & No & Manual & Yes* & Finitely many* \\
\hline Gogoi et al. [19] & 6 & No & Manual & Yes* & Finitely many* \\
\hline Sandhiya and Selvakumari [27] & 4 & No & Manual & Yes* & Finitely many* \\
\hline Borah et al. [29] & 3 & No & Manual & Yes $^{*}$ & Finitely many* \\
\hline Razak and Mohamad [30] & 8 & No & Manual & Yes $^{*}$ & 3 \\
\hline Proposed Algorithm 1/Example 10 & 19 & Yes & Manual \& Scilab codes & Yes & Finitely many \\
\hline
\end{tabular}

*: The number that can be calculated manually.

of Algorithm 1 are compared with those of the previous fuzzy soft decision making algorithms. In Table 2, we indicate that Algorithm 1 gives more satisfactory results than other decision making algorithms.

Note: During the calculations given in Table 2, in case of the presence of the weighted fuzzy soft sets, the inverse (weighted) fuzzy soft matrices corresponding to the respective sets were considered.

We present Tables 2 and 3 to demonstrate the advantages of IFSSPDM method (Algorithm 1).
Algorithm 1 also provides a solution for the decision making problems based on soft sets. Hence, we can compare our algorithm with the existing soft decision algorithms. In 2010, Çag̃man and Enginog̃lu [8] proposed the algorithms of SMmDM, SmMDM, SmmDM, and SMMDM to deal with a soft set based decision making. In 2018, Atagün et al. [9] stated that the products in these algorithms should be distributed in two directions. Thus, they introduced the algorithms of SDMmDM, SDmMDM, SDmmDM, and SDMMDM by revising the algorithms of Çağman and 
Enginog̃lu [8]. By solving the problem of decision making in Example 6 of Çag̃man and Enginog̃lu [8] using the SDMmDM algorithm, the optimum set is obtained as $\left\{u_{1}, u_{2}\right\}$. When the algorithms of FSMmDM, FSmMDM, FSMmDM-cw (see [28,31]), which are proposed to deal with the (weighted) fuzzy soft set based decision making, are recreated according to the idea of two- sided distribution of Atagün et al. [9], it is seen that the outputs of the recreated algorithms support the results of Algorithm 1. As the number of decision makers increases, it becomes difficult to produce an output with the manual steps of the algorithm proposed by Razak and Mohamad [30]. The optimum sets obtained using the SSRDM algorithm in [13] and Algorithm 1 coincide. Also, SSRDM algorithm has Scilab codes. However, the SSRDM is only proposed for MCGDM based on inverse soft sets (i.e., $\{0,1\}$ instead of $[0,1])$. The algorithms proposed in $[18,19,27,29]$ are insufficient to address the decision making problems involving different criterion sets and the calculations are performed manually. In summary, it can be said from Table 3 that each method compared to Algorithm 1 has its restrictions such as the number of decision makers, calculation strategy, and configuration of criteria. Algorithm 1 tackles to shortcomings of the existing methods and allows decision makers to freely choose without any restrictions.

\section{The IFSDIf-dDM method}

In this section, we propose a decision making algorithm called the Inverse Fuzzy Soft Distributive Ifdifference Decision Making (IFSDIf-dDM) algorithm (Algorithm 2), which produces a ranking order for the selection of objects of two discrete universal sets $O_{1}$ and $\mathrm{O}_{2}$ concerning the parameter set $X$. To apprehend Algorithm 2 precisely, a flow diagram (Figure 2) has been given. Also, we compare the results of the emerging algorithm with some of the existing fuzzy soft decision making algorithms.

\subsection{The algorithm of IFSDIf-dDM and its application}

Definition 22. Let $\left[a_{i j}^{\left(c, r_{1}\right)}\right] \in I F S M_{t \times v_{1}}$ and $\left[b_{i k}^{\left(c, r_{2}\right)}\right] \in$ $I F S M_{t \times v_{2}}$. Also, let $\left[a_{i j}^{\left(c, r_{1}\right)}\right] \asymp_{c}\left[b_{i k}^{\left(c, r_{2}\right)}\right]=\left[c_{i l}\right] \in$ IFSM $M_{t \times v_{1} v_{2}}$ :

(a) If $\left[a_{i j}^{\left(c, r_{1}\right)}\right] \Leftarrow_{c}\left[b_{i k}^{\left(c, r_{2}\right)}\right]=\left[g_{i l}\right] \in I F S M_{t \times v_{1} v_{2}}$ and $\left[g_{i l}\right] \sqcap\left[c_{i l}\right]=\left[e_{i l}\right] \in I F S M_{t \times v_{1} v_{2}}$, then the matrix $\left[\wp_{1 s}^{1}\right]$ is called a left-distributive left-if-difference decision inverse fuzzy soft matrix, where for each $s=1,2, \cdots, v_{1}$ :

$$
\wp_{1 s}^{1}=\frac{1}{v_{2}} \sum_{q=(s-1) v_{2}+1}^{s v_{2}} \beta_{1 q}, \quad\left(0 \leq \wp_{1 s}^{1} \leq 1\right),
$$

such that:

$$
\beta_{1 q}=\frac{1}{t+1}\left(\frac{1}{t} \sum_{i=1}^{t} g_{i q}+\sum_{i=1}^{t} e_{i q}\right) .
$$

(b) If $\left[a_{i j}^{\left(c, r_{1}\right)}\right] \Rightarrow_{c}\left[b_{i k}^{\left(c, r_{2}\right)}\right]=\left[h_{i l}\right] \in I F S M_{t \times v_{1} v_{2}}$ and $\left[h_{i l}\right] \sqcap\left[c_{i l}\right]=\left[f_{i l}\right] \in I F S M_{t \times v_{1} v_{2}}$, then the matrix $\left[\eta_{1 s}^{1}\right]$ is called a left-distributive right-if-difference decision inverse fuzzy soft matrix, where for each $s=1,2, \cdots, v_{1}$ :

$$
\eta_{1 s}^{1}=\frac{1}{v_{2}} \sum_{q=(s-1) v_{2}+1}^{s v_{2}} \delta_{1 q}, \quad\left(0 \leq \eta_{1 s}^{1} \leq 1\right)
$$

such that:

$$
\delta_{1 q}=\frac{1}{t+1}\left(\frac{1}{t} \sum_{i=1}^{t} h_{i q}+\sum_{i=1}^{t} f_{i q}\right) .
$$

Definition 23. Let $\left[a_{i j}^{\left(c, r_{1}\right)}\right] \in I F S M_{t \times v_{1}}$ and $\left[b_{i k}^{\left(c, r_{2}\right)}\right] \in$ $I F S M_{t \times v_{2}}$. Also, let $\left[b_{i k}^{\left(c, r_{2}\right)}\right] \asymp_{c}\left[a_{i j}^{\left(c, r_{1}\right)}\right]=\left[d_{i l}\right] \in$ IFSM $M_{t \times v_{1} v_{2}}$ :

(a) If $\left[b_{i k}^{\left(c, r_{2}\right)}\right] \Leftarrow_{c}\left[a_{i j}^{\left(c, r_{1}\right)}\right]=\left[g_{i l}^{\prime}\right] \in I F S M_{t \times v_{2} v_{1}}$ and $\left[g_{i l}^{\prime}\right] \sqcap\left[d_{i l}\right]=\left[e_{i l}^{\prime}\right] \in I F S M_{t \times v_{2} v_{1}}$, then the matrix $\left[\wp_{1 s}^{2}\right]$ is called a right-distributive left-if-difference decision inverse fuzzy soft matrix, where for each $s=1,2, \cdots, v_{2}$ :

$$
\wp_{1 s}^{2}=\frac{1}{v_{1}} \sum_{p=(s-1) v_{1}+1}^{s v_{1}} \beta_{1 p}, \quad\left(0 \leq \wp_{1 s}^{2} \leq 1\right)
$$

such that:

$$
\beta_{1 p}=\frac{1}{t+1}\left(\frac{1}{t} \sum_{i=1}^{t} g_{i p}^{\prime}+\sum_{i=1}^{t} e_{i p}^{\prime}\right)
$$

(b) If $\left[b_{i k}^{\left(c, r_{2}\right)}\right] \Rightarrow_{c}\left[a_{i j}^{\left(c, r_{1}\right)}\right]=\left[h_{i l}^{\prime}\right] \in I F S M_{t \times v_{2} v_{1}}$ and $\left[h_{i l}^{\prime}\right] \sqcap\left[d_{i l}\right]=\left[f_{i l}^{\prime}\right] \in I F S M_{t \times v_{2} v_{1}}$, then the matrix $\left[\eta_{1 s}^{2}\right]$ is called a right-distributive right-if-difference decision inverse fuzzy soft matrix, where for each $s=1,2, \cdots, v_{2}$ :

$$
\eta_{1 s}^{2}=\frac{1}{v_{1}} \sum_{p=(s-1) v_{1}+1}^{s v_{1}} \delta_{1 p}, \quad\left(0 \leq \eta_{1 s}^{2} \leq 1\right),
$$

such that:

$$
\delta_{1 p}=\frac{1}{t+1}\left(\frac{1}{t} \sum_{i=1}^{t} h_{i p}^{\prime}+\sum_{i=1}^{t} f_{i p}^{\prime}\right) .
$$

Definition 24. Let $\left[\wp_{1 s}^{1}\right],\left[\eta_{1 s}^{1}\right]$ be left-distributive left and right-If-difference decision inverse fuzzy soft matrices and $\left[\wp_{1 s}^{2}\right],\left[\eta_{1 s}^{2}\right]$ be right-distributive left and 


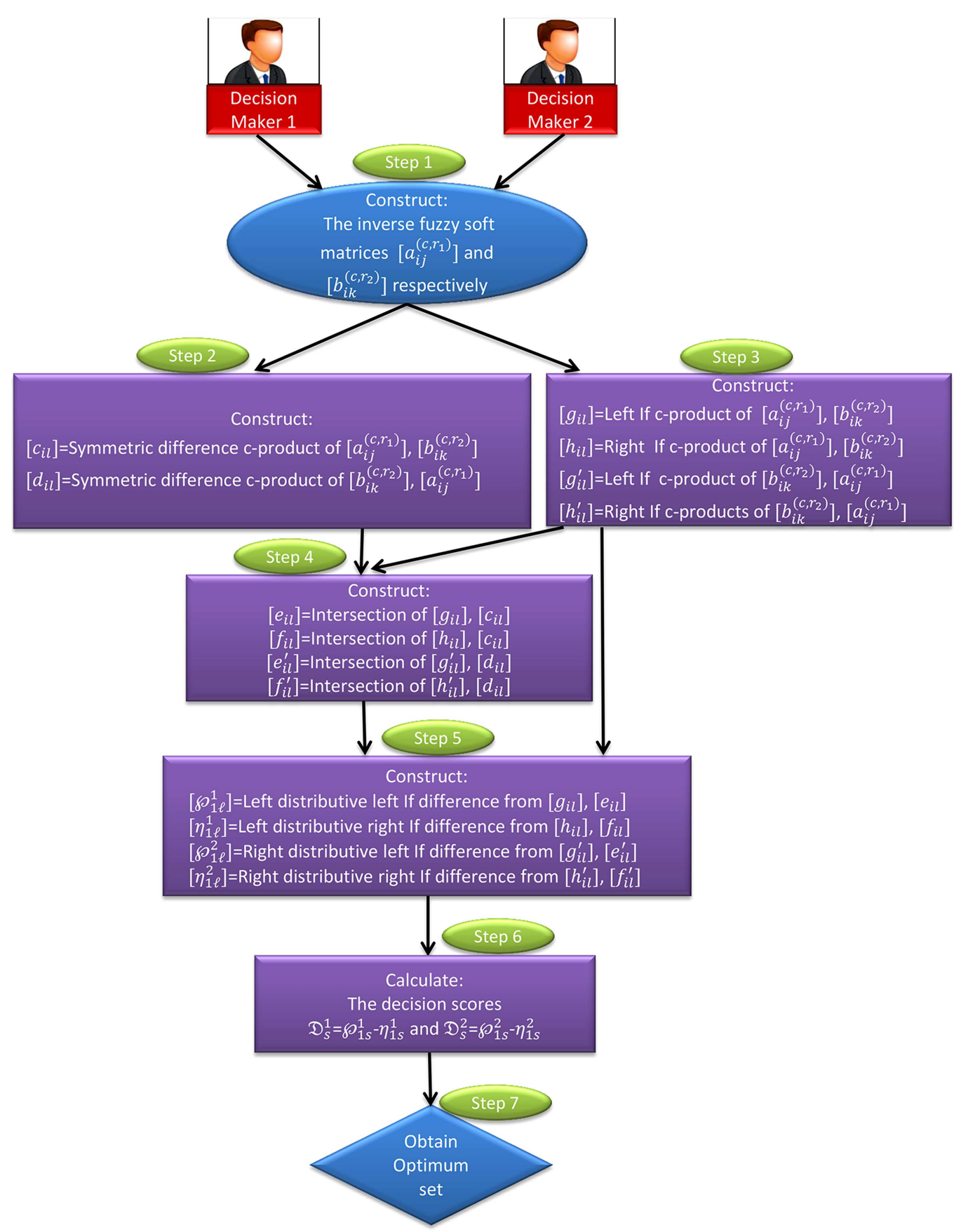

Figure 2. The flow diagram of Algorithm 2.

right-If-difference decision inverse fuzzy soft matrices,respectively. Then, the decision score of the sth object $\left(o_{s}^{r}\right)$ of the universal set $O_{r}(r=1,2)$ is defined and denoted by:

$$
\mathfrak{D}_{s}^{r}=\wp_{1 s}^{r}-\eta_{1 s}^{r} .
$$

Definition 25. Let $O_{1}=\left\{o_{1}^{1}, o_{2}^{1}, \cdots, o_{v_{1}}^{1}\right\}$ and $O_{2}=$ $\left\{o_{1}^{2}, o_{2}^{2}, \cdots, o_{v_{2}}^{2}\right\}$ be two discrete universal object sets. By using decision scores of objects of these universal sets, we get the ranking order of two objects $O_{s_{1}}^{r_{1}}$ and $o_{s_{2}}^{r_{2}}$ as follows:

$$
o_{s_{1}}^{r_{1}} \succ o_{s_{2}}^{r_{2}} \quad \text { if } \quad \mathfrak{D}_{s_{1}}^{r_{1}}>\mathfrak{D}_{s_{2}}^{r_{2}}
$$

where it can be $r_{1}=r_{2}$ or $r_{1} \neq r_{2}$, and $s_{1}=s_{2}$ or $s_{1} \neq$ $s_{2}$. Then, the optimum set of $O_{1}$ and $O_{2}$ is obtained as follows:

$$
\begin{gathered}
O p t_{\mathfrak{D}}\left(O_{1}, O_{2}\right)=\left\{o_{j}^{r_{1}}: o_{j}^{r_{1}} \in O_{1} \quad \text { or } \quad o_{j}^{r_{1}} \in O_{2}\right. \\
\text { and } \mathfrak{D}_{j}^{r_{1}}>\mathfrak{D}_{j^{\prime}}^{r_{1}} \text { for } \forall j^{\prime} \neq j \\
\text { and } \left.\mathfrak{D}_{j}^{r_{1}}>\mathfrak{D}_{j^{\prime}}^{r_{2}} \text { for } \forall j^{\prime}\right\} .
\end{gathered}
$$


Using the above concepts, we create the subsequent algorithm to search out the optimal choice for two inverse fuzzy soft sets over the discrete universal object set $O_{1}, O_{2}$ and a common parameter set $X$.

\section{Algorithm 2.}

Step 1. The experts create the inverse fuzzy soft matrices (represented as $\left[a_{i j}^{\left(c, r_{1}\right)}\right]$ and $\left[b_{i k}^{\left(c, r_{2}\right)}\right]$ ) corresponding to their inverse soft sets.

Step 2. Obtain the symmetric difference $c$-products $\left[c_{i l}\right]=\left[a_{i j}^{\left(c, r_{1}\right)}\right] \asymp_{c}\left[b_{i k}^{\left(c, r_{2}\right)}\right]$ and $\left[d_{i l}\right]=\left[b_{i k}^{\left(c, r_{2}\right)}\right] \asymp_{c}$ $\left[a_{i j}^{\left(c, r_{1}\right)}\right]$.

Step 3. Obtain the If $c$-products $\left[g_{i l}\right],\left[h_{i l}\right],\left[g_{i l}^{\prime}\right]$, and $\left[h_{i l}^{\prime}\right]$ as follows:

$$
\begin{aligned}
& {\left[g_{i l}\right]=\left[a_{i j}^{\left(c, r_{1}\right)}\right] \Leftarrow_{c}\left[b_{i k}^{\left(c, r_{2}\right)}\right],} \\
& {\left[h_{i l}\right]=\left[a_{i j}^{\left(c, r_{1}\right)}\right] \Rightarrow_{c}\left[b_{i k}^{\left(c, r_{2}\right)}\right],} \\
& {\left[g_{i l}^{\prime}\right]=\left[b_{i k}^{\left(c, r_{2}\right)}\right] \Leftarrow_{c}\left[a_{i j}^{\left(c, r_{1}\right)}\right],} \\
& {\left[h_{i l}^{\prime}\right]=\left[b_{i k}^{\left(c, r_{2}\right)}\right] \Rightarrow_{c}\left[a_{i j}^{\left(c, r_{1}\right)}\right] .}
\end{aligned}
$$

Step 4. Obtain the inverse fuzzy soft matrices $\left[e_{i l}\right]$, $\left[f_{i l}\right],\left[e_{i l}^{\prime}\right]$, and $\left[f_{i l}^{\prime}\right]$ as follows:

$$
\begin{array}{ll}
{\left[e_{i l}\right]=\left[g_{i l}\right] \sqcap\left[c_{i l}\right],} & {\left[f_{i l}\right]=\left[h_{i l}\right] \sqcap\left[c_{i l}\right],} \\
{\left[e_{i l}^{\prime}\right]=\left[g_{i l}^{\prime}\right] \sqcap\left[d_{i l}\right],} & {\left[f_{i l}^{\prime}\right]=\left[h_{i l}^{\prime}\right] \sqcap\left[d_{i l}\right] .}
\end{array}
$$

Step 5. Find the left-distributive left and right-Ifdifference decision inverse fuzzy soft matrices $\left[\wp_{1 \ell}^{1}\right]$ and $\left[\eta_{1 \ell}^{1}\right]$, and the right-distributive left and right-Ifdifference decision inverse fuzzy soft matrices $\left[\wp_{1 \ell}^{2}\right]$ and $\left[\eta_{1 \ell}^{2}\right]$, respectively.

Step 6. Calculate decision scores $\mathfrak{D}_{s}^{1}$ and $\mathfrak{D}_{s}^{2}$ for each object in the discrete universal sets $\mathrm{O}_{1}$ and $\mathrm{O}_{2}$.

Step 7. Obtain an optimum set $O p t_{\mathfrak{D}}\left(O_{1}, O_{2}\right)$ concerning the decision scores for the objects.

We give a fictitious example to illustrate the possible application of Algorithm 2.
Example 11. Suppose that a local food company operating in Turkey wants to get into a partnership with a selection of the best companies in the same industry in Spain or Italy. There are two discrete universal sets, each involving possible options to get into a partnership: $O_{1}=\left\{o_{1}^{1}, o_{2}^{1}\right\}$ is a set of food companies in Spain and $\mathrm{O}_{2}=\left\{o_{1}^{2}, o_{2}^{2}, o_{3}^{2}\right\}$ is a set of a food companies in Italy. A decision committee consisting of two experts is composed of the members $E_{1}$ and $E_{2} . E_{1}$ is a well-informed (Spanish) expert of the existing food companies in Spain. $E_{2}$ is a wellinformed (Italian) expert of the existing food companies in Italy. Therefore, the management of a food company in Turkey proposes that expert $E_{1}$ should evaluate the food companies in $O_{1}$ and expert $E_{2}$ should evaluate the food companies in $\mathrm{O}_{2}$ according to the parameters in the set $X_{1}=\left\{x_{1}^{1}\right.$ - environmental risks, $x_{2}^{1}$-social risks $x_{3}^{1}$ - political risks, $x_{4}^{1}$-financial fluctuations $x_{5}^{1}$-exchange ratefluctuations, $x_{6}^{1}$-cyclical fluctuations $\}$. After the experts create the inverse fuzzy soft sets, we are ready to apply Algorithm 2 to determining the optimal company in order to get into a partnership with the food company in Turkey.

Assume that the inverse fuzzy soft sets of the experts $E_{1}$ and $E_{2}$ are, respectively, shown in Box II. Now, we can use Algorithm 2.

Step 1. The inverse fuzzy soft matrices corresponding to the inverse fuzzy soft sets of experts $E_{1}$ and $E_{2}$ are respectively created as follows:

$$
\begin{aligned}
{\left[a_{i j}^{(1,1)}\right] } & =\left[\begin{array}{ll}
0.4 & 0.9 \\
0.6 & 0.7 \\
0.4 & 0.5 \\
0.3 & 0.6 \\
0.9 & 0.5 \\
0.1 & 0.8
\end{array}\right], \\
{\left[b_{i k}^{(1,2)}\right] } & =\left[\begin{array}{ccc}
0.1 & 0.9 & 0.1 \\
0.4 & 0.8 & 0.3 \\
0.2 & 0.8 & 0.2 \\
0.3 & 0 & 0.8 \\
0.2 & 0.7 & 0.3 \\
0.5 & 0.8 & 1
\end{array}\right] .
\end{aligned}
$$

$$
\begin{aligned}
& \tilde{\Psi}_{O_{1}}^{X_{1}}=\left\{\begin{array}{l}
\left(o_{1}^{1},\left\{\left(x_{1}^{1}, 0.4\right),\left(x_{2}^{1}, 0.6\right),\left(x_{3}^{1}, 0.4\right),\left(x_{4}^{1}, 0.3\right),\left(x_{5}^{1}, 0.9\right),\left(x_{6}^{1}, 0.1\right)\right\}\right), \\
\left(o_{2}^{1},\left\{\left(x_{1}^{1}, 0.9\right),\left(x_{2}^{1}, 0.7\right),\left(x_{3}^{1}, 0.5\right),\left(x_{4}^{1}, 0.6\right),\left(x_{5}^{1}, 0.5\right),\left(x_{6}^{1}, 0.8\right)\right\}\right)
\end{array}\right\} \\
& \tilde{\Psi}_{O_{2}}^{X_{1}}=\left\{\begin{array}{l}
\left(o_{1}^{2},\left\{\left(x_{1}^{1}, 0.1\right),\left(x_{2}^{1}, 0.4\right),\left(x_{3}^{1}, 0.2\right),\left(x_{4}^{1}, 0.3\right),\left(x_{5}^{1}, 0.2\right),\left(x_{6}^{1}, 0.5\right)\right\}\right), \\
\left(o_{2}^{2},\left\{\left(x_{1}^{1}, 0.9\right),\left(x_{2}^{1}, 0.8\right),\left(x_{3}^{1}, 0.8\right),\left(x_{4}^{1}, 0\right),\left(x_{5}^{1}, 0.7\right),\left(x_{6}^{1}, 0.8\right)\right\}\right), \\
\left(o_{3}^{2},\left\{\left(x_{1}^{1}, 0.1\right),\left(x_{2}^{1}, 0.3\right),\left(x_{3}^{1}, 0.2\right),\left(x_{4}^{1}, 0.8\right),\left(x_{5}^{1}, 0.3\right),\left(x_{6}^{1}, 1\right)\right\}\right)
\end{array}\right\}
\end{aligned}
$$


Step 2. By using the symmetric difference c-products of inverse fuzzy soft matrices, we obtain:

$$
\begin{aligned}
{\left[c_{i l}\right] } & =\left[a_{i j}^{(1,1)}\right] \asymp_{c}\left[b_{i k}^{(1,2)}\right] \\
& =\left[\begin{array}{cccccc}
0.3 & 0.5 & 0.3 & 0.8 & 0 & 0.8 \\
0.2 & 0.2 & 0.3 & 0.3 & 0.1 & 0.4 \\
0.2 & 0.4 & 0.2 & 0.3 & 0.3 & 0.3 \\
0 & 0.3 & 0.5 & 0.3 & 0.6 & 0.2 \\
0.7 & 0.2 & 0.6 & 0.3 & 0.2 & 0.2 \\
0.4 & 0.7 & 0.9 & 0.3 & 0 & 0.2
\end{array}\right], \\
{\left[d_{i l}\right] } & =\left[\begin{array}{cccccc}
b_{i k}^{(1,2)} & \asymp_{c} & {\left[a_{i j}^{(1,1)}\right.}
\end{array}\right] \\
& =\left[\begin{array}{cccccc}
0.3 & 0.8 & 0.5 & 0 & 0.3 & 0.8 \\
0.2 & 0.3 & 0.2 & 0.1 & 0.3 & 0.4 \\
0.2 & 0.3 & 0.4 & 0.3 & 0.2 & 0.3 \\
0 & 0.3 & 0.3 & 0.6 & 0.5 & 0.2 \\
0.7 & 0.3 & 0.2 & 0.2 & 0.6 & 0.2 \\
0.4 & 0.3 & 0.7 & 0 & 0.9 & 0.2
\end{array}\right] .
\end{aligned}
$$

Step 3. By employing the If c-products of inverse fuzzy soft matrices, the inverse fuzzy soft matrices are obtained. $\left[g_{i l}\right]=\left[a_{i j}^{(1,1)}\right] \Leftarrow_{c}\left[b_{i k}^{(1,2)}\right],\left[h_{i l}\right]=$ $\left[a_{i j}^{(1,1)}\right] \Rightarrow_{c}\left[b_{i k}^{(1,2)}\right]$ and $\left[g_{i l}^{\prime}\right]=\left[b_{i k}^{(1,2)}\right] \Leftarrow_{c}\left[a_{i j}^{(1,1)}\right]$, $\left[h_{i l}^{\prime}\right]=\left[b_{i k}^{(1,2)}\right] \Rightarrow_{c}\left[a_{i j}^{(1,1)}\right]$ are respectively obtained as follows:

$$
\begin{aligned}
& {\left[g_{i l}\right]=\left[\begin{array}{llllll}
1 & 0 & 1 & 1 & 1 & 1 \\
1 & 0 & 1 & 1 & 0 & 1 \\
1 & 0 & 1 & 1 & 0 & 1 \\
1 & 1 & 0 & 1 & 1 & 0 \\
1 & 1 & 1 & 1 & 0 & 1 \\
0 & 0 & 0 & 1 & 1 & 0
\end{array}\right],} \\
& {\left[h_{i l}\right]=\left[\begin{array}{llllll}
0 & 1 & 0 & 0 & 1 & 0 \\
0 & 1 & 0 & 0 & 1 & 0 \\
0 & 1 & 0 & 0 & 1 & 0 \\
1 & 0 & 1 & 0 & 0 & 1 \\
0 & 0 & 0 & 0 & 1 & 0 \\
1 & 1 & 1 & 0 & 1 & 1
\end{array}\right],}
\end{aligned}
$$

and

$$
\begin{aligned}
& {\left[g_{i l}^{\prime}\right]=\left[\begin{array}{llllll}
0 & 0 & 1 & 1 & 0 & 0 \\
0 & 0 & 1 & 1 & 0 & 0 \\
0 & 0 & 1 & 1 & 0 & 0 \\
1 & 0 & 0 & 0 & 1 & 1 \\
0 & 0 & 0 & 1 & 0 & 0 \\
1 & 0 & 1 & 1 & 1 & 1
\end{array}\right],} \\
& {\left[h_{i l}^{\prime}\right]=\left[\begin{array}{llllll}
1 & 1 & 0 & 1 & 1 & 1 \\
1 & 1 & 0 & 0 & 1 & 1 \\
1 & 1 & 0 & 0 & 1 & 1 \\
1 & 1 & 1 & 1 & 0 & 0 \\
1 & 1 & 1 & 0 & 1 & 1 \\
0 & 1 & 0 & 1 & 0 & 0
\end{array}\right] .}
\end{aligned}
$$

Step 4. Through the intersection operation, the following inverse fuzzy soft matrices are obtained:

$$
\begin{aligned}
{\left[e_{i l}\right] } & =\left[g_{i l}\right] \sqcap\left[c_{i l}\right] \\
& =\left[\begin{array}{cccccc}
0.3 & 0 & 0.3 & 0.8 & 0 & 0.8 \\
0.2 & 0 & 0.3 & 0.3 & 0 & 0.4 \\
0.2 & 0 & 0.2 & 0.3 & 0 & 0.3 \\
0 & 0.3 & 0 & 0.3 & 0.6 & 0 \\
0.7 & 0.2 & 0.6 & 0.3 & 0 & 0.2 \\
0 & 0 & 0 & 0.3 & 0 & 0
\end{array}\right], \\
{\left[f_{i l}\right] } & =\left[\begin{array}{cccccc}
\left.h_{i l}\right] & \sqcap\left[c_{i l}\right] & & & & \\
0 & 0.5 & 0 & 0 & 0 & 0 \\
0 & 0.2 & 0 & 0 & 0.1 & 0 \\
0 & 0.4 & 0 & 0 & 0.3 & 0 \\
0 & 0 & 0.5 & 0 & 0 & 0.2 \\
0 & 0 & 0 & 0 & 0.2 & 0 \\
0.4 & 0.7 & 0.9 & 0 & 0 & 0.2
\end{array}\right],
\end{aligned}
$$

and

$$
\begin{aligned}
& {\left[e_{i l}^{\prime}\right]=\left[g_{i l}^{\prime}\right] \sqcap\left[d_{i l}\right]} \\
& =\left[\begin{array}{cccccc}
0 & 0 & 0.5 & 0 & 0 & 0 \\
0 & 0 & 0.2 & 0.1 & 0 & 0 \\
0 & 0 & 0.4 & 0.3 & 0 & 0 \\
0 & 0 & 0 & 0 & 0.5 & 0.2 \\
0 & 0 & 0 & 0.2 & 0 & 0 \\
0.4 & 0 & 0.7 & 0 & 0.9 & 0.2
\end{array}\right], \\
& {\left[f_{i l}^{\prime}\right]=\left[h_{i l}^{\prime}\right] \sqcap\left[d_{i l}\right]} \\
& =\left[\begin{array}{cccccc}
0.3 & 0.8 & 0 & 0 & 0.3 & 0.8 \\
0.2 & 0.3 & 0 & 0 & 0.3 & 0.4 \\
0.2 & 0.3 & 0 & 0 & 0.2 & 0.3 \\
0 & 0.3 & 0.3 & 0.6 & 0 & 0 \\
0.7 & 0.3 & 0.2 & 0 & 0.6 & 0.2 \\
0 & 0.3 & 0 & 0 & 0 & 0
\end{array}\right] .
\end{aligned}
$$

Step 5. The left-distributive left and right-Ifdifference decision inverse fuzzy soft matrices are:

$$
\left[\wp_{1 s}^{1}\right]=\left[\begin{array}{ll}
0.2444 & 0.3222
\end{array}\right]
$$

and

$$
\left[\eta_{1 s}^{1}\right]=\left[\begin{array}{ll}
0.2349 & 0.1031
\end{array}\right]
$$

The right-distributive left and right-If-difference decision inverse fuzzy soft matrices are:

$$
\left[\wp_{1 s}^{2}\right]=\left[\begin{array}{lll}
0.0523 & 0.2785 & 0.1761
\end{array}\right],
$$

and

$$
\left[\eta_{1 s}^{2}\right]=\left[\begin{array}{lll}
0.3952 & 0.1380 & 0.3166
\end{array}\right]
$$


Step 6. The decision score for each object in the discrete universal sets $\mathrm{O}_{1}$ and $\mathrm{O}_{2}$ is calculated as follows:

$$
\begin{aligned}
& \mathfrak{D}_{1}^{1}=0.0095, \quad \mathfrak{D}_{2}^{1}=0.2191, \quad \mathfrak{D}_{1}^{2}=-0.3429, \\
& \mathfrak{D}_{2}^{2}=0.1405, \quad \text { and } \quad \mathfrak{D}_{3}^{2}=-0.1405 .
\end{aligned}
$$

Step 7. Then, we obtain the ranking order of objects in $\mathrm{O}_{1}$ and $\mathrm{O}_{2}$ as:

$$
o_{2}^{1} \succ o_{2}^{2} \succ o_{1}^{1} \succ o_{3}^{2} \succ o_{1}^{2},
$$

since:

$$
\mathfrak{D}_{2}^{1}>\mathfrak{D}_{2}^{2}>\mathfrak{D}_{1}^{1}>\mathfrak{D}_{3}^{2}>\mathfrak{D}_{1}^{2} .
$$

So, the optimum set of $O_{1}$ and $O_{1}$ is $\mathrm{Opt}_{\mathfrak{D}}\left(O_{1}, O_{2}\right)=$ $\left\{o_{2}^{1}\right\}$.

Therefore, according to the data presented by the experts $E_{1}$ and $E_{2}$, the food company in Turkey can get into apartnership with the food company $o_{2}^{1}$ (in Spain).

\subsection{Advantages and limitations of the IFSDIf-dDM method (Algorithm 2) in comparison}

Consider any two inverse fuzzy soft matrices consisting of two disjoint discrete universal sets with a common parameter set. By nature, these two inverse fuzzy soft matrices can be merged to form a brand new inverse fuzzy soft matrix. It lets us find the optimal choice for Example 11 by the existing Multi-Criteria Decision Making (MCDM) strategies on fuzzy soft sets after converting the inverse fuzzy soft matrix into to fuzzy soft matrix. On the other hand, any inverse fuzzy soft matrix, which has more than one column, can be bifurcated.

Hence, we can apply our proposed IFSDIf-dDM (Algorithm 2) method in order to find the optimal preference for the MCDM problems under the surroundings fuzzy soft sets after remodeling the fuzzy soft matrix into the inverse fuzzy soft matrix. The herbal merging and bifurcation lead us to compare our proposed IFSDIf-dDM method (Algorithm 2) with some existing MCDM strategies for fuzzy soft sets. Moreover, since fuzzy soft sets are the generalized shape of soft sets, we will examine our proposed IFSDIf-dDM approach (Algorithm 2) with some current MCDM techniques on soft sets. Example 12 illustrated the ideas of merging and bifurcation of inverse fuzzy soft matrices.
Example 12. Consider the inverse fuzzy soft matrices $\left[a_{i j}^{(1,1)}\right]$ and $\left[b_{i k}^{(1,2)}\right]$ given in Example 11. Note that those two inverse fuzzy soft matrices are produced from the two discrete universal sets $O_{1}$ and $O_{2}$ respectively over a commonplace parameter set $X$. Therefore, they can be merged evidently to form a brand new inverse fuzzy soft matrix as follows:

$$
\begin{aligned}
\operatorname{Merge}\left(\left[a_{i j}^{(1,1)}\right],\left[b_{i k}^{(1,2)}\right]\right) & =\left[\begin{array}{ccccc}
0.4 & 0.9 & 0.1 & 0.9 & 0.1 \\
0.6 & 0.7 & 0.4 & 0.8 & 0.3 \\
0.4 & 0.5 & 0.2 & 0.8 & 0.2 \\
0.3 & 0.6 & 0.3 & 0 & 0.8 \\
0.9 & 0.5 & 0.2 & 0.7 & 0.3 \\
0.1 & 0.8 & 0.5 & 0.8 & 1
\end{array}\right] \\
& =\widetilde{\Psi}_{X}^{\text {merge }\left(O_{1}, O_{2}\right)} .
\end{aligned}
$$

Moreover, the merged inverse fuzzy matrix, i.e., $\widetilde{\Psi}_{X}^{\text {merge }\left(O_{1}, O_{2}\right)}$ can be bifurcated as follows:

$\left[\begin{array}{lll}0.4 & 0.9 & 0.1 \\ 0.6 & 0.7 & 0.4 \\ 0.4 & 0.5 & 0.2 \\ 0.3 & 0.6 & 0.3 \\ 0.9 & 0.5 & 0.2 \\ 0.1 & 0.8 & 0.5\end{array}\right] \quad$ and $\quad\left[\begin{array}{cc}0.9 & 0.1 \\ 0.8 & 0.3 \\ 0.8 & 0.2 \\ 0 & 0.8 \\ 0.7 & 0.3 \\ 0.8 & 1\end{array}\right]$

We report that the number of bifurcations of an inverse fuzzy soft matrix with $n$ columns is $n-1$. It leads to defining the Order of Bifurcation (OB) and the Ordinate's Difference from the Order of Bifurcation (ODOB) as follows.

Definition 26. Let $A=\left[a_{i j}\right]_{m \times n}$ be an inverse fuzzy soft matrix. Assume that $A$ is bifurcated as $B=$ $\left[b_{i j}\right]_{m \times n_{1}}$ and $C=\left[c_{i j}\right]_{m \times n_{2}}$. Then, $O B=\left(n_{1}, n_{2}\right)$ and $O D O B=n_{1}-n_{2}$. For instance, in Example 12, $O B=(3,2)$ and $O D O B=1$.

Suppose that the number of columns of an inverse fuzzy soft matrix is an odd (even) number, then the different values of ODOB are odd (even) numbers. Different values of ODOB (either odd or even numbers) influence the ranking order of our proposed IFSDIfdDM method (Algorithm 2). To show this, we consider the inverse fuzzy soft matrix $\widetilde{\Psi}_{X}^{\text {merge }\left(O_{1}, O_{2}\right)}$ (Example 12) in Table 4 and the inverse fuzzy soft matrix $\left[a_{m j}^{(1,1)}\right]$ (Example 10) in Table 5.

Maji et al. [35] proposed the choice value method and Roy and Maji [16] proposed the comparison value

Table 4. The influence of ODOB (odd numbers) on the ranking of the IFSDIf-dDM method (Algorithm 2).

\begin{tabular}{cccccccc}
\hline $\boldsymbol{O B}$ & $\boldsymbol{O D} \boldsymbol{O B}$ & \multicolumn{5}{c}{ Decision score } & Ranking order \\
\hline$(1,4)$ & -3 & -0.0630952 & 0.2809524 & -0.2142857 & 0.2333333 & -0.047619 & $o_{2}^{1} \succ o_{2}^{2} \succ o_{3}^{2} \succ o_{1}^{1} \succ o_{1}^{2}$ \\
$(2,3)$ & -1 & 0.0095238 & 0.2190476 & -0.3428571 & 0.1404762 & -0.1404762 & $o_{2}^{1} \succ o_{2}^{2} \succ o_{1}^{1} \succ o_{3}^{2} \succ o_{1}^{2}$ \\
$(3,2)$ & 1 & -0.0928571 & 0.0928571 & -0.3071429 & 0.2349206 & -0.0301587 & $o_{2}^{2} \succ o_{2}^{1} \succ o_{3}^{2} \succ o_{1}^{1} \succ o_{1}^{2}$ \\
$(4,1)$ & 3 & 0.047619 & 0.2333333 & -0.1904762 & 0.2333333 & -0.0809524 & $o_{2}^{1}=o_{2}^{2} \succ o_{1}^{1} \succ o_{3}^{2} \succ o_{1}^{2}$ \\
\hline
\end{tabular}


Table 5. The influence of ODOB (even numbers) on the ranking of IFSDIf-dDM method (Algorithm 2).

\begin{tabular}{lllllllll}
\hline $\boldsymbol{O B}$ & $\boldsymbol{O D O B}$ & \multicolumn{9}{c}{ Decision score } & Ranking order \\
\hline$(1,5)$ & -4 & -0.075 & 0.1583333 & 0.3416667 & -0.2083333 & 0.1083333 & -0.025 & $o_{3}^{1} \succ o_{2}^{1} \succ o_{5}^{1} \succ o_{6}^{1} \succ o_{1}^{1} \succ o_{4}^{1}$ \\
$(2,4)$ & -2 & -0.0541667 & 0.0625 & 0.3041667 & -0.2458333 & 0.0291667 & -0.1041667 & $o_{3}^{1} \succ o_{2}^{1} \succ o_{5}^{1} \succ o_{1}^{1} \succ o_{6}^{1} \succ o_{4}^{1}$ \\
$(3,3)$ & 0 & 0.0416667 & 0.1722222 & 0.3277778 & -0.2916667 & -0.0861111 & -0.1638889 & $o_{3}^{1} \succ o_{2}^{1} \succ o_{1}^{1} \succ o_{5}^{1} \succ o_{6}^{1} \succ o_{4}^{1}$ \\
$(4,2)$ & 2 & -0.0416667 & 0.1166667 & 0.3 & -0.1666667 & -0.0270833 & -0.0770833 & $o_{3}^{1} \succ o_{2}^{1} \succ o_{5}^{1} \succ o_{1}^{1} \succ o_{6}^{1} \succ o_{4}^{1}$ \\
$(5,1)$ & 4 & 0.025 & 0.1833333 & 0.2833333 & -0.1833333 & -0.0333333 & -0.055 & $o_{3}^{1} \succ o_{2}^{1} \succ o_{1}^{1} \succ o_{5}^{1} \succ o_{6}^{1} \succ o_{4}^{1}$ \\
\hline
\end{tabular}

technique to sort the alternatives under the environment of soft sets and fuzzy soft sets, respectively. Kong et al. [17] commented on and revised the comparison value technique of Roy and Maji [16]. Liu et al. [22] proposed the hamming distance value approach for fuzzy soft sets. Our proposed IFSDIf-dDM approach (Algorithm 2) dominates these techniques: choice value, comparison value, revised comparison value, and hamming distance value. Petchimuthu and Kamac1 [36] proposed the inverse soft distributive If-sum decision making method to handle the problem consisting of two disjoint universal sets with a common parameter set under the environment of inverse soft sets. It produced equal effects in examination with the proposed IFSDIfdDM method (Algorithm 2). In comparison with the MCDM strategies of Xiao [26] (belief entropy with Dempster- Shafer (D-S) evidence theory), Li et al. [24] (grey relational analysis and D-S theory of evidence), Wang et al. [25] (ambiguity measure and D-S theory of evidence), Basu et al. [23] (mean potentiality approach), our proposed IFSDIf-dDM technique (Algorithm 2) produces the same or better outcomes. The whole discussion of the comparisons is given in Table 6 .

\subsubsection{The advantages of the IFSDIf-dDM method (Algorithm 2)}

(i) Our proposed IFSDIf-dDM approach (Algorithm 2) may be applied in order to find the ranking order of MCGDM problems consisting of two discrete universal sets over a common parameter set;

(ii) Since a soft set may be considered as a fuzzy soft set or a fuzzy soft set may be considered as an inverse fuzzy soft set, our proposed IFSDIf-dDM approach (Algorithm 2) can be employed to solve MCDM issues in an environment of soft sets and fuzzy soft sets through a bifurcation of inverse fuzzy soft sets. It produces more convincing results than most of the present MCDM methods. It is shown in Table 6;

(iii) By employing the Scilab codes created for our proposed IFSDIf-dDM technique (Algorithm 2), satisfactory results can be achieved quickly without human error.

\subsubsection{Limitations of the IFSDIf-dDM method (Algorithm 2)}

When making a bifurcation of a matrix corresponding to a soft set, fuzzy soft set, or inverse fuzzy soft set (i.e., creating two new matrices from one matrix by separating its columns), ODOB has to be selected carefully; because ODOB affects the ranking order of objects, as shown in Tables 4 and 5 . The maximum preferable consideration of ODOB is that $O D O B=-1(O D O B=-2)$ for odd (even) numbers of $O D O B$. It is shown in Table 6 .

\section{Conclusion}

In this study, we introduced the concepts of inverse fuzzy soft matrices and addressed various products on them. We proved some propositions including associative commutative laws for the emerging products. Also, we constructed two different Multi-Criteria Group Decision Making (MCGDM) models employing the inverse fuzzy soft matrix products. We stated that these models could achieve more precise calculations and produce more accurate results than some of the current fuzzy soft decision models.

The inverse fuzzy soft matrices representing two different types of inverse fuzzy soft sets provided a two-way progress, practicality, and efficiency during calculations. The adaptability of matrix operations to programming languages greatly affected the computation time and thereby, the practicality of the logical operations was supported. Another advantage of inverse fuzzy soft matrices was producing solutions for decision making problems that involved both multiple-discrete parameter sets and two discrete universal object sets. By considering the practicality and usefulness of inverse fuzzy soft matrices, we hope that new uses can be introduced for them so that the approaches to dealing with decision making involving multiple-discrete universal object sets can be proposed. Also, the findings about the operations of the inverse fuzzy soft matrices and their algebraic structures can be enhanced. The 
Table 6. Comparison of the IFSDIf-dDM method (Algorithm 2) with other methods.

\begin{tabular}{|c|c|c|c|c|}
\hline Problem & Method & Ranking order & $\begin{array}{l}\text { Proposed } \\
\text { method }\end{array}$ & $\begin{array}{c}\text { Ranking order } \\
\text { of proposed } \\
\text { method }\end{array}$ \\
\hline Example $12\left(\widetilde{\Psi}_{X}^{\operatorname{merge}\left(O_{1}, O_{2}\right)}\right)$ & $\begin{array}{l}\text { Maji et al. [35] } \\
\text { Roy and Maji [16] } \\
\text { Kong et al. [17] }\end{array}$ & $\begin{array}{l}o_{2}^{1}=o_{2}^{2} \succ o_{1}^{1}=o_{3}^{2} \succ o_{1}^{2} \\
o_{2}^{1}=o_{2}^{2} \succ o_{1}^{1} \succ o_{3}^{2} \succ o_{1}^{2} \\
o_{2}^{1}=o_{2}^{2} \succ o_{1}^{1}=o_{3}^{2} \succ o_{1}^{2}\end{array}$ & $\begin{array}{c}\text { Algorithm } 2 \\
(O D O B=-1)\end{array}$ & $o_{2}^{1} \succ o_{2}^{2} \succ o_{1}^{1} \succ o_{3}^{2} \succ o_{1}^{2}$ \\
\hline Table 2 in [22] & Liu et al. [22] & $\begin{array}{c}x_{6} \succ x_{4} \succ x_{3} \succ x_{7} \\
\succ x_{2} \succ x_{5}=x_{8} \succ x_{1}\end{array}$ & $\begin{array}{c}\text { Algorithm } 2 \\
(O D O B=-2)\end{array}$ & $\begin{array}{c}u_{6} \succ u_{4} \succ u_{3} \succ u_{7} \\
\succ u_{2} \succ u_{5} \succ u_{8} \succ u_{1}\end{array}$ \\
\hline $\begin{array}{l}\text { Example } 5.5 \text { (Step 3) in [36] } \\
\text { Example } 5.6(\text { Step } 3) \text { in }[36]\end{array}$ & $\begin{array}{c}\text { Petchimuthu } \\
\text { and Kamacı [36] }\end{array}$ & $\begin{array}{c}u_{1}^{2} \succ u_{3}^{1} \succ u_{1}^{1} \succ u_{2}^{2} \succ u_{2}^{1} \\
u_{2}^{1} \succ u_{2}^{2} \succ u_{3}^{1} \succ u_{4}^{1} \succ u_{1}^{1} \succ u_{1}^{2}\end{array}$ & Algorithm 2 & $\begin{array}{c}u_{1}^{2} \succ u_{3}^{1} \succ u_{1}^{1} \succ u_{2}^{2} \succ u_{2}^{1} \\
u_{2}^{1} \succ u_{2}^{2} \succ u_{3}^{1} \succ u_{4}^{1} \succ u_{1}^{1} \succ u_{1}^{2}\end{array}$ \\
\hline Table 2 in [24] & $\begin{array}{l}\text { Xiao [26] } \\
\text { Khameneh et al. [21] } \\
\text { Wang et al. [25] }\end{array}$ & $\begin{array}{l}x_{2} \succ x_{3} \succ x_{1} \\
x_{2} \succ x_{3} \succ x_{1} \\
x_{2} \succ x_{3} \succ x_{1}\end{array}$ & $\begin{array}{c}\text { Algorithm } 2 \\
(O D O B=-1)\end{array}$ & $x_{2} \succ x_{3} \succ x_{1}$ \\
\hline Table 14 in [26] & $\begin{array}{l}\text { Basu et al. [23] } \\
\text { Xiao [26] } \\
\text { Li et al. [24] }\end{array}$ & $\begin{array}{l}x_{3} \succ x_{1}=x_{4} \succ x_{2} \\
x_{3} \succ x_{1} \succ x_{4} \succ x_{2} \\
x_{3} \succ x_{1} \succ x_{4} \succ x_{2}\end{array}$ & $\begin{array}{l}\text { Algorithm } 2 \\
(O D O B=-2)\end{array}$ & $x_{3} \succ x_{1} \succ x_{4} \succ x_{2}$ \\
\hline Table 10 in [25] & $\begin{array}{l}\text { Wang et al. [25] } \\
\text { Li et al. [24] }\end{array}$ & $\begin{array}{l}x_{3} \succ x_{1} \succ x_{4} \succ x_{2} \\
x_{3} \succ x_{4} \succ x_{1} \succ x_{2}\end{array}$ & $\begin{array}{c}\text { Algorithm } 2 \\
(O D O B=-2)\end{array}$ & $x_{3} \succ x_{4} \succ x_{1} \succ x_{2}$ \\
\hline
\end{tabular}

concept of inversing, which gives bidirectionality to the soft approach function, can be adopted for most of the fuzzy hybridizations of soft sets. In further research, it is necessary and meaningful to develop the inverse structures of intuitionistic fuzzy sets, picture fuzzy sets, and neutrosophic sets. We will focus on the issues mentioned above in the future.

\section{Acknowledgments}

The authors are deeply grateful to the editor and reviewers for their suggestions to improve the quality of the paper.

\section{References}

1. Zadeh. L.A. "Fuzzy sets", Information and Control, 8(3), pp. 338-353 (1965).

2. Molodtsov, D. "Soft set theory-first results", Computers and Mathematics with Applications, 37(4-5), pp. 19-31 (1999).
3. Maji, P.K., Biswas. R., and Roy, A.R. "Soft set theory", Computers and Mathematics with Applications, 45(4-5), pp. 555-562 (2003).

4. Ali, M.I., Feng, F., Liu, X., et al. "On some new operations in soft set theory", Computers and Mathematics with Applications, 57(9), pp. 1547-1553 (2009).

5. Alkhazaleh, S., Salleh, A.R., and Hassan, N. "Soft multisets theory", Applied Mathematical Sciences, 5(72), pp. 3561-3573 (2011).

6. Han, B.H. and Geng, S.L. "Pruning method for optimal solutions of $i n t^{m}-i n t^{n}$ decision making scheme", European Journal of Operational Research, 231(3), pp. 779-783 (2013).

7. Çag̃man, N. and Enginog̃lu, S. "Soft set theory and uni-int decision making", European Journal of Operational Research, 207(2), pp. 848-855 (2010).

8. Çag̃man, N. and Enginog̃lu, S. "Soft matrix theory and its decision making", Computers and Mathematics with Applications, 59(10), pp. 3308-3314 (2010).

9. Atagün, A.O., Kamacı, H., and Oktay, O. "Reduced soft matrices and generalized products with appli- 
cations in decision making", Neural Computing and Applications, 29(9), pp. 445-456 (2018).

10. Basu, T.M., Mahapatra, N.M., and Mondal, S.K. "Matrices in soft set theory and their applications in decision making problems", South Asian Journal of Mathematics, 2(2), pp. 126-143 (2012).

11. Kamacı, H., Atagün, A.O., and Sönmezog̃lu, A. "Rowproducts of soft matrices with applications in multipledisjoint decision making", Applied Soft Computing, 62, pp. 892-914 (2018).

12. Kamacı, H., Atagün, A.O., and Toktaş, E. "Bijective soft matrix theory and multi-bijective linguistic soft decision system", Filomat, 32(11), pp. 3799-3814 (2018).

13. Kamacı, H., Saltık, K., Akız, H.F., et al. "Cardinality inverse soft matrix theory and its applications in multicriteria group decision making", Journal of Intelligent and Fuzzy Systems, 34(3), pp. 2031-2049 (2018).

14. Kamacı, H., Atagün, A.O., and Aygün, E. "Difference operations of soft matrices with applications in decision making", Punjab University Journal of Mathematics, 51(3), pp. 1-21 (2019).

15. Maji, P.K., Biswas, R., and Roy, A.R. "Fuzzy soft sets", Journal of Fuzzy Mathematics 9(3), pp. 589-602 (2001).

16. Roy, A.R. and Maji, P.K. "A fuzzy soft set theoretic approach to decision making problems", Journal of Computational and Applied Mathematics, 203(2), pp. 412-418 (2007).

17. Kong, Z., Gao, L., and Wang, L. "Comment on a fuzzy soft set theoretic approach to decision making problems", Journal of Computational and Applied Mathematics, 223(2), pp. 540-542 (2009).

18. Das, P.K. and Borgohain, R. "An application of fuzzy soft set in multicriteria decision making problem", International Journal of Computer Applications, 38(12), pp. 33-37 (2012).

19. Gogoi, K., Dutta, A.Kr., and Chutia, C. "Application of fuzzy soft set theory in day to day problems", International Journal of Computer Applications, 85(7), pp. 27-31 (2014).

20. Eraslan, S. "A decision making method via TOPSIS on fuzzy soft sets", Journal of New Results in Science, 4(8), pp. $57-70$ (2015).

21. Khameneh, A.Z., Kılıçman, A., and Salleh, A.R. "An adjustable approach to multi-criteria group decisionmaking based on a preference relationship under fuzzy soft information", International Journal of Fuzzy Systems, 19(6), pp. 1840-1865 (2017).

22. Liu, Z., Qin, K., and Pei, Z. "A method for fuzzy soft sets in decision-making based on an ideal solution", Symmetry, 9(10), p. 246 (2017).

23. Basu, T.M., Mahapatra, N.K., and Mondal, S.K. "A balanced solution of a fuzzy soft set based decision making problem in medical science", Applied Soft Computing, 12(10), pp. 3260-3275 (2012).
24. Li, Z., Wen, G., and Xie, N. "An approach to fuzzy soft sets in decision making based on grey relational analysis and Dempster-Shafer theory of evidence: An application in medical diagnosis", Artificial Intelligence in Medicine, 64(3), pp. 161-171 (2015).

25. Wang, J., Hu, Y., Xiao, F., et al. "A novel method to use fuzzy soft sets in decision making based on ambiguity measure and Dempster-Shafer theory of evidence: an application in medical diagnosis", Artificial Intelligence in Medicine, 69, pp. 1-11 (2016).

26. Xiao, F. "A hybrid fuzzy soft sets decision making method in medical diagnosis", IEEE Access, 6, pp. 25300-25312 (2018).

27. Sandhiya, S. and Selvakumari, K. "Application of fuzzy soft sets in job requirement problem", Journal of Computer and Mathematical Sciences, 10(1), pp. 184-189 (2019).

28. Çag̃man, N. and Enginog̃lu, S. "Fuzzy soft matrix theory and its application in decision making", Iranian Journal of Fuzzy Systems, 9(1), pp. 109-119 (2012).

29. Borah, M.J., Neog, T.J., and Sut, D.K. "Fuzzy soft matrix theory and its decision making", International Journal of Modern Engineering Research, 2(2), pp. 121-127 (2012).

30. Razak, S.A. and Mohamad, D. "A decision making method using fuzzy soft sets", Malaysian Journal of Fundamental and Applied Sciences, 9(2), pp. 99-104 (2013).

31. Inthumathi, V., Chitra, V., and Jayasree, S. "Fuzzy soft min-max decision making and its applications", Journal of Informatics and Mathematical Sciences, 9(3), pp. 827-834 (2017).

32. Razak, S.A., Mohamad, D., and Abdullah, I.I. "A group decision making problem using hierarchical based fuzzy soft matrix approach", International Journal of Advanced and Applied Sciences, 4(10), pp. 26-32 (2017).

33. Çetkin, V., Aygünog̃lu, A., and Aygün, H. "A new approach in handling soft decision making problems", Journal of Nonlinear Science and Applications, 9, pp. 231-239 (2016).

34. Khalil, A.M. and Hassan, N. "Inverse fuzzy soft set and its application in decision making", International Journal of Information and Decision Sciences, 11(1), pp. 73-92 (2019).

35. Maji, P.K., Roy, A.R., and Biswas, R. "An application of soft sets in a decision making problem", Comput. Math. Appl., 44, pp. 1077-1083 (2002).

36. Petchimuthu, S. and Kamacı, H. "The row-products of inverse soft matrices in multicriteria decision making", Journal of Intelligent and Fuzzy Systems, 36(6), pp. 6425-6441 (2019).

\section{Appendix}

Scilab codes for Algorithms 1 and 2 are generated in Figure A.1. 


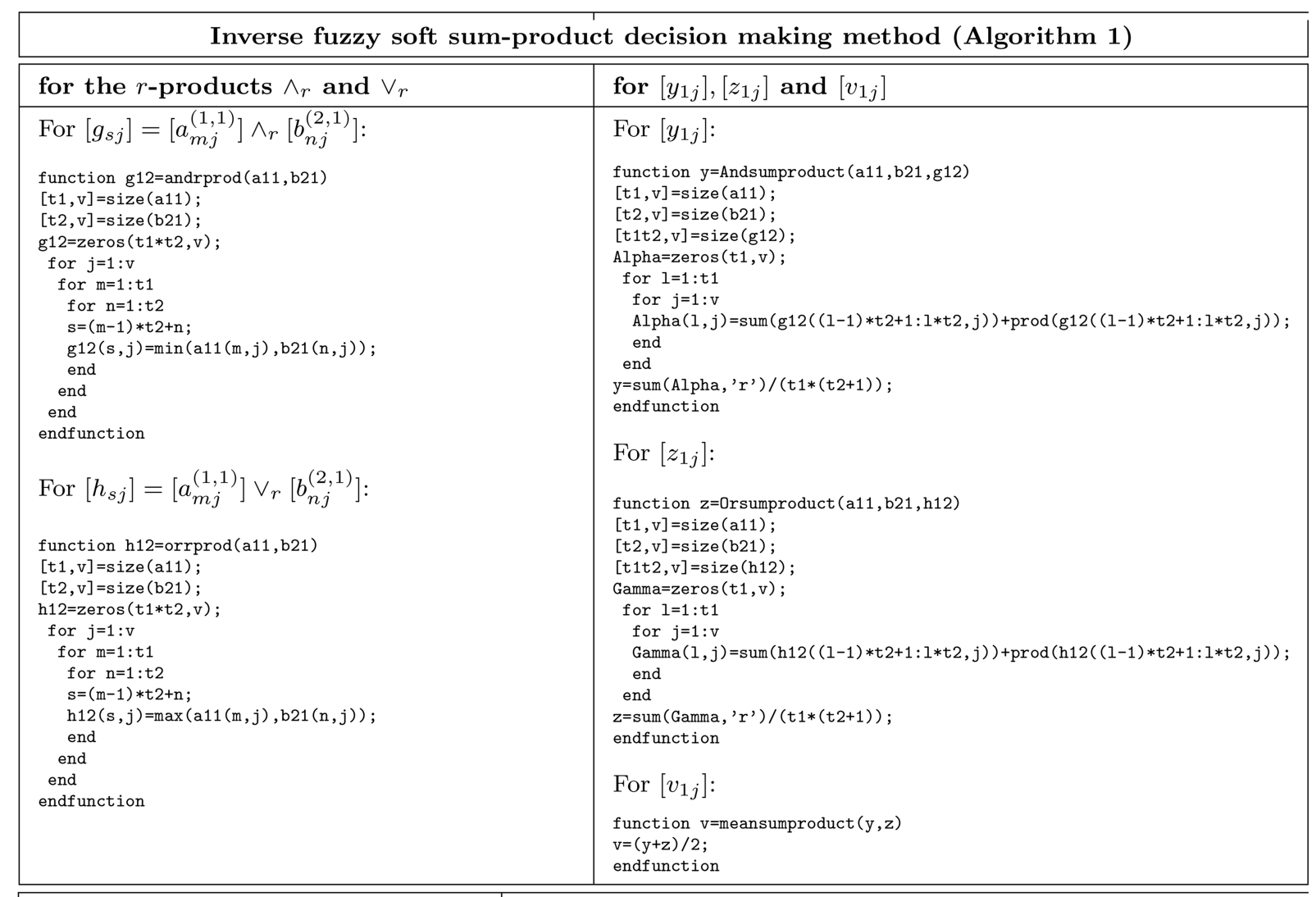

Inverse fuzzy soft distributive If-difference decision making method (Algorithm 2)

\begin{tabular}{|c|c|c|}
\hline for the $c$-product $\asymp_{c}$ & for the $c$-products $\Leftarrow_{c}$ and $\Rightarrow_{c}$ & for the intersection $\sqcap$ \\
\hline 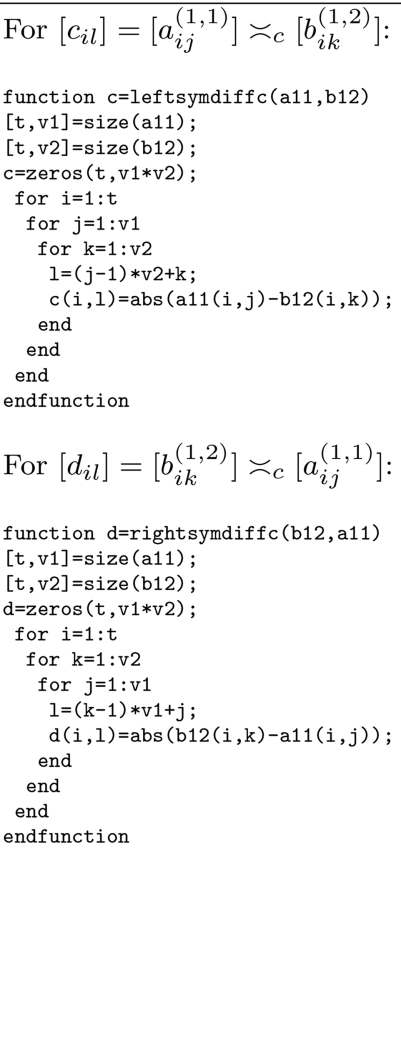 & 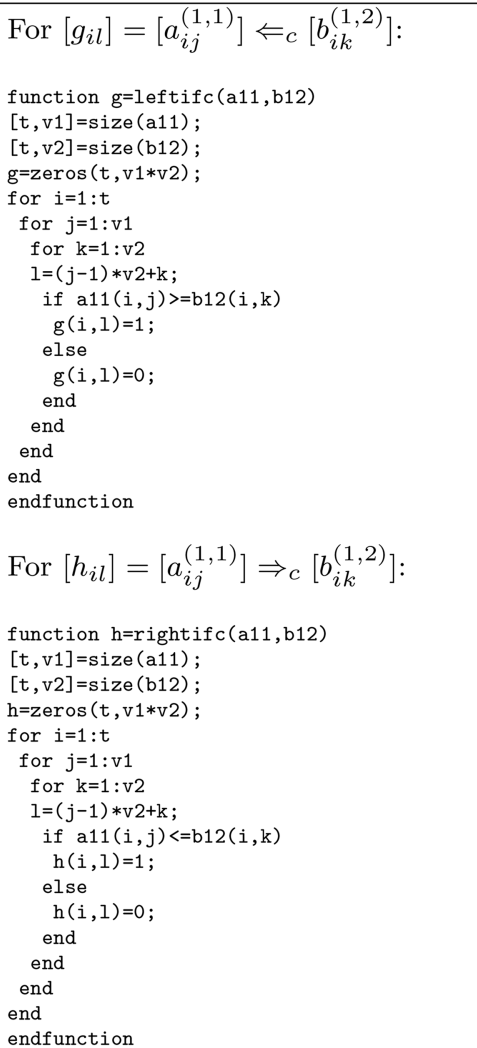 & 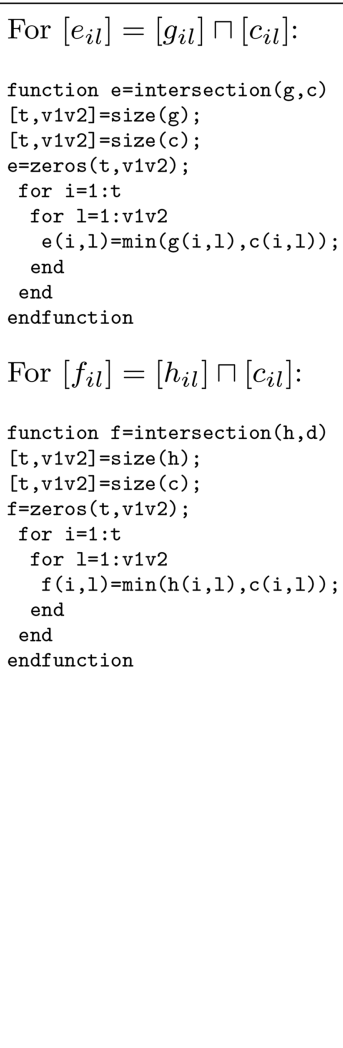 \\
\hline
\end{tabular}

Figure A.1. Scilab codes for Algorithms 1 and 2. 


\begin{tabular}{|c|c|c|}
\hline for the matrices $\left[\wp_{1 s}^{1}\right]$ and $\left[\eta_{1 s}^{1}\right]$ & for the matrices $\left[\wp_{1 s}^{2}\right]$ and $\left[\eta_{1 s}^{2}\right]$ & for decision score $\mathfrak{D}_{s}^{r}$ \\
\hline 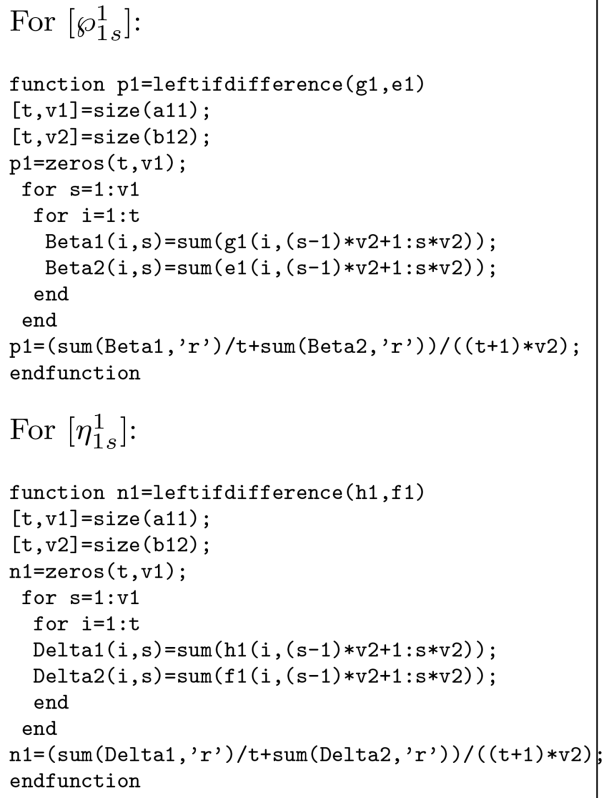 & 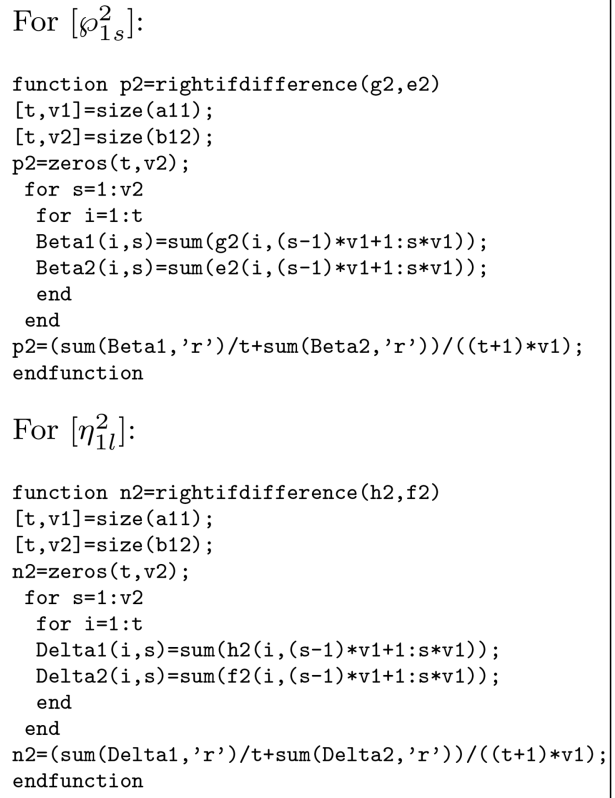 & 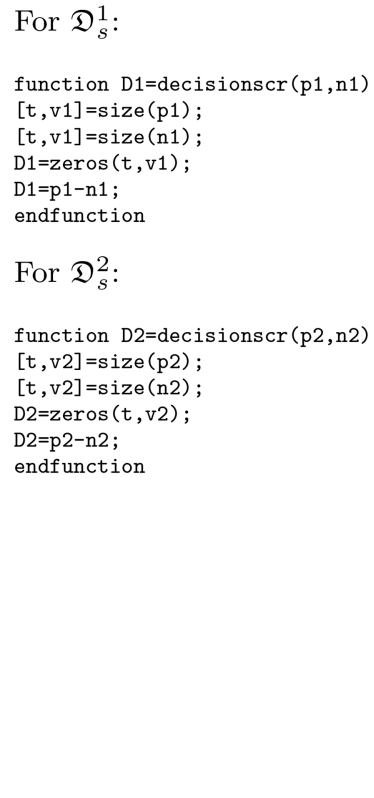 \\
\hline
\end{tabular}

Figure A.1. Scilab codes for Algorithms 1 and 2 (continued).

\section{Biographies}

Subramanian Petchimuthu is an Assistant Professor in the Department of Mathematics at University College of Engineering Nagercoil (a Constituent College of Anna University, Chennai), Kanyakumari district, Tamilnadu, India. He received his MSc degree from Manonmaniam Sundaranar University, Tamilnadu, in 2005 and $\mathrm{PhD}$ degree from Periyar University, Tamilnadu, in 2009. His main areas of research interests are group theory, ring theory, nearring, gamma ring theory, soft sets, fuzzy sets, and multi-criteria group decision making problems.

Hüseyin Kamacı is an Associate Professor in the Mathematics Department in the Science and Arts Faculty of Yozgat Bozok University, Turkey. He received his $\mathrm{MSc}$ and $\mathrm{PhD}$ degrees in Mathematics from Bozok University, Yozgat, Turkey, in 2014 and 2018, respectively. His research interests include mathematical logic, set theory, operational research, computational intelligence, and decision making. He has many valuable publications on these issues in different scientific journals. 